\title{
Quarkonia and Quark Drip Lines in Quark-Gluon Plasma
}

\author{
Cheuk-Yin Wong \\ Physics Division, Oak Ridge National Laboratory, Oak Ridge, TN 37831 and \\ Department of Physics, University of Tennessee, Knoxville, TN 37996
}

(Dated: January 7, 2018)

\begin{abstract}
We extract the $Q-\bar{Q}$ potential by using the thermodynamic quantities obtained in lattice gauge calculations. The potential is tested and found to give spontaneous dissociation temperatures that agree well with those from lattice gauge spectral function analysis. Using such a $Q-\bar{Q}$ potential, we examine the quarkonium states in a quark-gluon plasma and determine the 'quark drip lines' which separate the region of bound color-singlet $Q \bar{Q}$ states from the unbound region. The characteristics of the quark drip lines severely limit the region of possible bound $Q \bar{Q}$ states with light quarks to temperatures close to the phase transition temperature. Bound quarkonia with light quarks may exist very near the phase transition temperature if their effective quark mass is of the order of 300-400 MeV and higher.
\end{abstract}

PACS numbers: 25.75.-q 25.75.Dw

\section{INTRODUCTION}

The degree to which the constituents of a quark-gluon plasma (QGP) can combine to form composite entities is an important property of the plasma. It has significant implications on the nature of the phase transition, the quark-gluon plasma equation of state, the probability of recombination of plasma constituents prior to the phase transition, and the chemical yields of the observed bound hadrons. The successes of the recombination model 1 ,

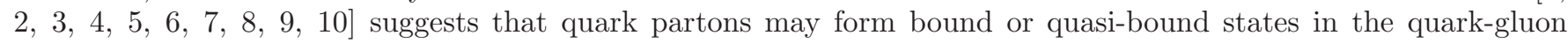
plasma, at least at, or close to, the phase transition temperature. It is an important theoretical question as to the range of temperatures in which these quarkonia may be bound or quasi-bound. The successes of the thermal model $[1,2,3,4,11,12,13,14,15,16,17,18,19,20]$ for hadron yields also raises the important question whether hadrons may become bound or quasi-bound in the quark-gluon plasma. If they are indeed bound in the quark-gluon plasma, the approach to chemical equilibrium may commence in the quark-gluon plasma phase before the phase transition and the boundaries of the quark-gluon plasma phase and the hadron phase may overlap.

Recent spectral analyses of quarkonium correlators indicated that $J / \psi$ may be stable up to $1.6 T_{c}$ where $T_{c}$ is the phase transition temperature [21, 22]. Subsequently, there has been renewed interest in quarkonium states in quark-gluon plasma as Zahed and Shuryak suggested that $Q \bar{Q}$ states with light quarks may be bound up to a few $T_{c}$ [23]. Quarkonium bound states and instanton molecules in the quark-gluon plasma have been considered by Brown, Lee, Rho, and Shuryak 24]. As heavy quarkonia may be used as a diagnostic tool 25], there have been many recent investigations on the stability of heavy quarkonia in the plasma 26, 26, 27, 28, 29, 30, 31, 32, 33, 34, 35, 36, 37, 38, 39].

Previously, DeTar [40], Hansson, Lee, and Zahed [41], and Simonov 42] observed that the range of strong interaction is not likely to change drastically across the phase transition and suggested the possible existence of relatively narrow low-lying $Q \bar{Q}$ states in the plasma. On the other hand, Hatsuda and Kunihiro [43] considered the persistence of soft modes in the plasma which may manifest themselves as pion-like and sigma-like states. The use of baryonstrangeness correlation and charge fluctuation to study the abundance of light quarkonium states in the plasma have been suggested recently [44, 45].

We would like to investigate the composite properties of the plasma and to determine its 'quark drip lines'. We shall focus our attention on color-singlet $Q-\bar{Q}$ states and the $Q-\bar{Q}$ quantities in this paper refer to those of color-singlet $Q-\bar{Q}$ states unless specified otherwise. Here we follow Werner and Wheeler [46] and use the term 'drip line' to separate the region of bound color-singlet $Q \bar{Q}$ states from the unbound region of spontaneous quarkonium dissociation. It should be emphasized that a quarkonium can be dissociated by collision with constituent particles to lead to the corresponding 'particle-dissociation lines', which can be interesting subjects for future investigation.

We would like to use the potential model to study the stability of quarkonia, as the potential model can be used to evaluate many more quantities than the lattice gauge spectral function analysis. The potential model lends itself to extrapolation into unknown regions of quark masses and temperatures. An important physical quantity in the potential model is the $Q-\bar{Q}$ potential between the quark $Q$ and the antiquark $\bar{Q}$ at a separation $R$ at a temperature $T$. Previous work in the potential model uses the color-singlet free energy $F_{1}(R, T)$ [26, 31, 38] or the color-singlet internal energy $U_{1}(R, T)[23,32$, 37] obtained in lattice gauge calculations as the $Q-\bar{Q}$ potential, without rigorous theoretical justifications. Here, the subscripts of $U_{1}(R, T)$ and $F_{1}(R, T)$ refer to the color-singlet property of the $Q-\bar{Q}$ system. The internal energy $U_{1}(R, T)$ is significantly deeper and spatially more extended than the free energy $F_{1}(R, T)$. The degree of quarkonium binding will be significantly different whether one uses the internal energy $U_{1}(R, T)$ or the 
free energy $F_{1}(R, T)$ as the $Q-\bar{Q}$ potential. Treating the internal energy $U_{1}(R, T)$ as the $Q-\bar{Q}$ potential led Shuryak and Zahed to suggest the possibility of color-singlet quarkonium states with light quarks in the plasma [23]. The conclusions will be quite different if one uses the free energy $F_{1}(R, T)$ as the $Q-\bar{Q}$ potential.

While $F_{1}(R, T)$ or $U_{1}(R, T)$ can both be used as the $Q-\bar{Q}$ potential at $T=0$ (at which $F_{1}(R, T)=U_{1}(R, T)$ ), the situation is not so clear in a thermalized quark-gluon plasma. It is important to find out the meaning of these thermodynamical quantities calculated in the finite-temperature lattice gauge theory so as to extract the $Q-\bar{Q}$ potential.

If one constructs the Schrödinger equation for the $Q$ and $\bar{Q}$ in a thermal medium, the $Q-\bar{Q}$ potential in the Schrödinger equation contains those interactions that act on $Q$ and $\bar{Q}$, when the medium particles have re-arranged themselves self-consistently. On the other hand, the total internal energy $U_{1}(R, T)$ contains not only these interactions that act on $Q$ and $\bar{Q}$, but also the internal gluon energy $U_{g}(R, T)$ relative to the gluon internal energy $U_{g 0}$ in the absence of $Q$ and $\bar{Q}$, as shown deductively in Ref. 27] starting from the definition of the free energy in lattice gauge theory in quenched QCD. If the gluon internal energy $U_{g}(R, T)$ were independent of $R$, then $U_{1}(R)$ could well be used as the $Q-\bar{Q}$ potential. However, in the grand canonical ensemble, $U_{g}(R, T)$ depends on $R$. To get the $Q-\bar{Q}$ potential, it is therefore necessary to subtract $U_{g}(R, T)-U_{g 0}$ from $U_{1}(R, T)$. As the subtleties of these results may not appear evident and the problem of non-perturbative QCD so intrinsically complicated, a thorough understanding of an analogous, but not identical, problem in QED is worth having. Therefore, we examine in detail the simple QED case of Debye screening of charges $Q$ and $\bar{Q}$ in a massless charged medium in a grand canonical ensemble, where the results can be readily obtained analytically. We would like to show that there is a relationship between the $Q-\bar{Q}$ potential and the total internal energy when screening occurs: the Debye screening $Q-\bar{Q}$ potential between two static opposite charges in QED can be obtained from the total internal energy by subtracting out the internal energy of the medium particles.

The results in the Debye screening case in QED support our previous conclusion in Ref. [27] that in the QCD lattice gauge calculations in the grand canonical ensemble, it is necessary to subtract out the $R$-dependent internal energy of the QGP from the total internal energy in order to obtain the potential between $Q$ and $\bar{Q}$ in the plasma. Additional lattice gauge calculations may be needed to evaluate the QGP internal energy in the presence of $Q$ and $\bar{Q}$. It is nonetheless useful at this stage to suggest approximate ways to evaluate the QGP internal energy. We proposed earlier a method by making use of the equation of state of the quark-gluon plasma obtained in an independent lattice gauge calculation 27]. The equation of state provides a relationship between the QGP internal energy and the QGP entropy content. As the QGP entropy content is the difference $U_{1}-F_{1}$, the $Q-\bar{Q}$ potential can be represented as a linear combination of $U_{1}$ and $F_{1}$, with coefficients depending on the quark-gluon plasma equation of state. The proposed potential was tested and found to give spontaneous dissociation temperatures that agree well with those from lattice gauge spectral function analysis in the quenched approximation.

The comparison of the potential model results with those from spectral analyses in the same quenched approximation is useful as a theoretical test of the potential model. However, in the quenched approximation, the quark-gluon plasma is assumed to consist of gluons only and the effects of dynamical quarks have not been included. As dynamical quarks provide additional screening, one wishes to know whether this additional screening will modify the binding energies of quarkonia significantly or not. The presence of dynamical quarks also lowers the phase transition temperature from $269 \mathrm{MeV}$ for quenched QCD to $154 \mathrm{MeV}$ for full QCD with three flavors [47]. For these reasons, it is necessary to include dynamical quarks to assess their effects on the stability of quarkonia. The knowledge of the single-particle states using potentials extracted from lattice gauge calculations in full QCD can then be used to examine the stability of both heavy and light quarkonia and to determine the location of the quark drip lines. We focus our attention mainly on heavy quarkonia for which a non-relativistic treatment is a good description. However, the problem of the stability of quarkonia with light quarks is intrinsically so complicated and the question of their stability up to a temperature of few units of $T_{c}$ so important [1]- 23] that even an approximate estimate using the non-relativistic potential model is worth having. The subject of light quarkonia will be examined again, with the inclusion of the relativistic effects as in recent works [48, 49], in the course of time.

The authors of Refs. [50, 51] claim recently that potential models cannot describe heavy quarkonia above $T_{c}$, as their potential model correlators fail to reproduce lattice gauge correlators for all cases with all types of potentials. Such a complete disagreement for all cases and all types of potentials suggests that the lack of agreement may not be due to the potential model (or models) themselves but to their method of evaluating the meson correlators in the potential model. We show recently that when the contributions from the bound states and continuum states are properly treated [30], the potential model correlators obtained with the proposed potential in Ref. [27] are consistent with lattice gauge correlators.

This paper is organized as follows. In Section II, we describe the puzzling behavior of the increase of the entropy of the QCD medium as the separation between $Q$ and $\bar{Q}$ increases. To understand such a behavior, we introduce in Section III a simple model of Debye screening in QED for which various thermodynamic quantities can be readily calculated. In Section VI, the variation of the number density and entropy density of the medium particles for a $Q-\bar{Q}$ pair in Debye screening is shown to depend on the $Q-\bar{Q}$ separation $R$ when the second-order contributions are included. In Section 
$\mathrm{V}$, we find similarly that the internal energy of the medium particles in Debye screening also increases with $R$ when second-order contributions are included. In Section VI, we examine the Schrödinger equation for the relative motion of $Q$ and $\bar{Q}$ and identify the potential between $Q$ and $\bar{Q}$ in Debye screening. We reach the conclusion that in order to obtain the Debye screening potential between two static charges in the grand canonical ensemble, it is necessary to subtract out the internal energy of the medium particles from the total internal energy. Returning to lattice gauge calculations in Section VII, we suggest a method in which the medium internal energy can be approximately determined and subtracted by using the information on the quark-gluon plasma of state. Consequently, the $Q-\bar{Q}$ potential turns out to be a linear combination of $U_{1}(R, T)$ and $F_{1}(R, T)$, with coefficients depending on the equation of state. In Section VIII, we use different potentials to calculate the spontaneous dissociation temperatures for various quarkonia and compare them with those obtained from lattice gauge spectral function analyses in quenched QCD. The dissociation temperatures obtained with the proposed linear combination of $U_{1}$ and $F_{1}$ give the best agreement with those from the spectral function analyses. In Section IX, we show how the thermodynamical quantities in full QCD with two flavors are parametrized. The dissociation temperatures for various heavy quarkonia for full QCD with two flavors are obtained in Section X. We introduce the quark drip lines in quark-gluon plasma in Section XI. We conclude and summarize our discussions in Section XII.

\section{THERMODYNAMICAL QUANTITIES IN LATTICE GAUGE CALCULATIONS AND DEBYE SCREENING}

Thermodynamical quantities for a heavy quark pair in the color-singlet state was studied by Kaczmarek and Zantow in quenched QCD and in full QCD with two flavors [33, 34]. They calculated $\left\langle\operatorname{tr} L(\mathbf{r} / 2) L^{\dagger}(-\mathbf{R} / 2)\right\rangle$ and obtained the color-singlet free energy $F_{1}(\mathbf{R}, T)$ from

$$
\left\langle\operatorname{tr} L(\mathbf{R} / 2) L^{\dagger}(-\mathbf{R} / 2)\right\rangle=e^{-F_{1}(\mathbf{R}, T) / k T} .
$$

Here $\operatorname{tr} L(\mathbf{R} / 2) L^{\dagger}(-\mathbf{R} / 2)$ is the trace of the product of two Polyakov lines at $\mathbf{R} / 2$ and $-\mathbf{R} / 2$. The free energy $F_{1}(\mathbf{R}, T)$, in the presence of the $Q-\bar{Q}$ pair, is measured relative to the free energy without the $Q-\bar{Q}$ pair. The quark and the antiquark lines do not, in general, form a close loop. As a gauge transformation introduces phase factors at the beginning and the end of an open Polyakov line, $\left\langle\operatorname{tr} L(\mathbf{R} / 2) L^{\dagger}(-\mathbf{R} / 2)\right\rangle$ is not gauge invariant under a gauge transformation. Calculations have been carried out in the Coulomb gauge which is the proper gauge to study bound states.

From the free energy $F_{1}$, Kaczmarek and Zantow [33, 34] calculated the internal energy $U_{1}$ using the statistical identity

$$
U_{1}(R, T)=F_{1}(R, T)+T S_{1}(R, T),
$$

where $S_{1}(R, T)=-\partial F_{1}(R, T) / \partial T$ is the entropy of the system in the presence of a color-singlet $Q-\bar{Q}$ pair and is measured relative to the entropy of the system in the absence of the $Q-\bar{Q}$ pair.

In order to extract the $Q-\bar{Q}$ potential from thermodynamical quantities calculated in the lattice gauge theory, we need to understand the behavior of the free energy $F_{1}(R, T)$, the internal energy $U_{1}(R, T)$, and $T$ times the entropy, $T S_{1}(R, T)$, which we shall also abbreviatingly call "the entropy" for simplicity of nomenclature. As these three quantities are related by Eq. (2) we need to find out the behavior of only two of these three quantities. Following the terminology used in lattice gauge calculations [34], $U_{1}, F_{1}$, and $T S_{1}$ are defined as being measured relative to their corresponding quantities in the absence of $Q$ and $\bar{Q}$.

We can begin by studying the entropy $T S_{1}(R, T)$ of the system. We note that the lattice gauge calculations show that the total entropy $T S_{1}(R)$ increases as a function of $R$, and saturates after the separation reaches a large value of $R$ [34] as shown in Fig. 1(a). What does such a behavior tell us about the response of the medium particles to the presence of the external color sources of $Q$ and $\bar{Q}$ in a thermal bath?

In the system under consideration, the system consists of the $Q$, the antiquark $\bar{Q}$ and the quark-gluon plasma. For simplicity we can examine the quenched case for which the quark-gluon plasma is assumed to consist of gluons only. The entropy of the system therefore comes from the sum of the entropies of $Q, \bar{Q}$ and the gluons. However, in the lattice gauge calculations in a thermal bath, the $Q$ and the $\bar{Q}$ are held fixed and do not contribute to the entropy of the system. The entropy of the system $T S_{1}$ comes entirely from the gluons. By fixing a temperature and focusing our attention at the state of thermal equilibrium in lattice gauge calculations, the gluons are in a grand canonical ensemble in contact with the thermal bath. The content and thermodynamical properties of the gluons are determined by the condition of thermal equilibrium at the fixed temperature of the thermal bath. The observed behavior of the entropy $T S_{1}(R)$ as a function of $R$ in Fig. 1(a) suggests that the gluon entropy content increases as the separation $R$ increases, and the entropy saturates when $R$ reaches a certain limit. 

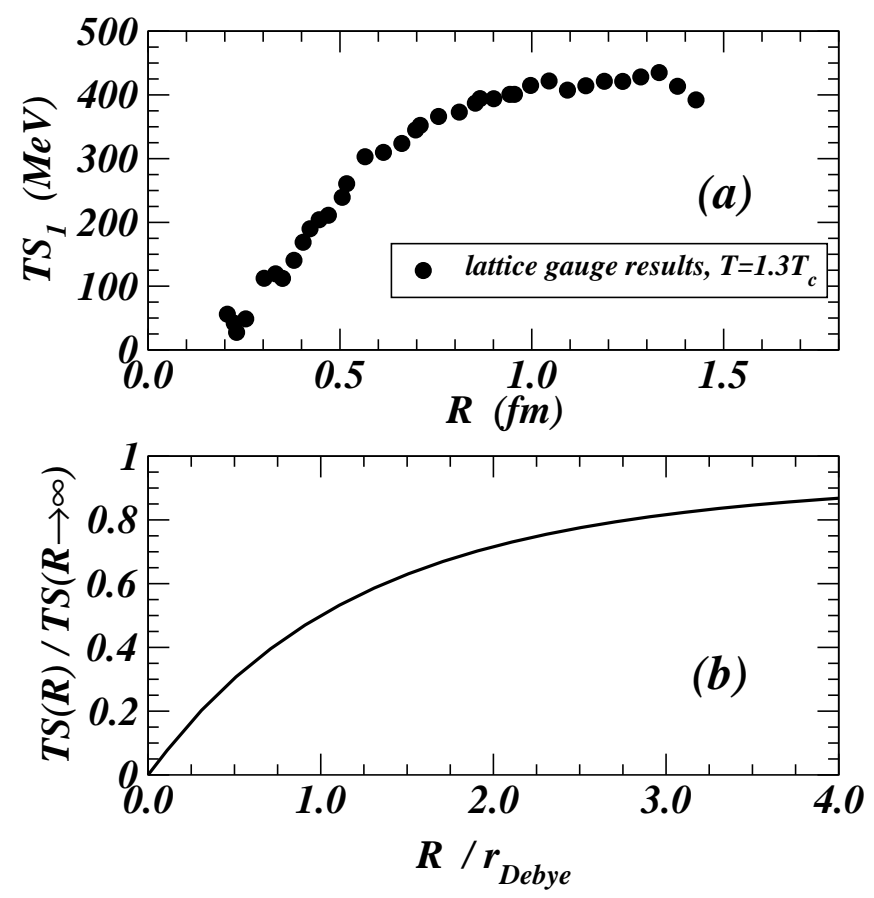

FIG. 1: (a) The total entropy $T S_{1}(R, T)$ as a function of $R$ at $T=1.3 T_{c}$ from the lattice gauge calculations [34]. (b) The ratio $\eta(R)=T S(R) / T S(R \rightarrow \infty)$ (which is also equal to $N(R) / N(R \rightarrow \infty)$ and $U(R) / U(R \rightarrow \infty)$ ) as a function of $R / r_{D}$ in a thermal medium under Debye screening.

As we know from the work of Landau and Belenkii [52], the entropy content of a system is closely correlated with the number content of the particles in the system. The behavior of the entropy suggests that the number of gluons increases as the separation $R$ increases. How do we understand such a behavior? If the number of gluons increases as a function of $R$, what happens to the internal energy content of the gluons as a function of $R$, and how does that affect the total internal energy and the potential between $Q$ and $\bar{Q}$ we wish to extract?

\section{ANALOGOUS PROBLEM OF DEBYE SCREENING IN QED}

The QCD problem of a quark and an antiquark in the presence of the actions of the gluons is so complicated that it is worth having even a good understanding of the puzzling behavior in an analogous, but not identical, problem of a static positive charge $Q$ and another negative charge $\bar{Q}$ under the action of charged medium particles in QED. We would like to ask whether there is a similar behavior of the number and the entropy of medium particles as the separation $R$ between the positive charge $Q$ and the negative charge $\bar{Q}$ increases. If the number of medium particles increases as a function of $R$, what happens to the internal energy content of the system and the medium particles as a function of $R$, and what is the relationship between the potential between $Q$ and $\bar{Q}$ and total internal energy we wish to extract?

Accordingly, we study the simple system of Debye screening and consider a $Q$ with charge $+q$ held fixed at $-\mathbf{R} / 2$ and a $\bar{Q}$ with a charge $-q$ at $\mathbf{R} / 2$, in a medium of massless fermions with charges $e_{ \pm}= \pm q$, in a thermal bath of temperature $T$ in a grand canonical ensemble. Any pair of charged particles with charges $e_{1}$ and $e_{2}$ separated by a distance $\mathbf{r}$ are assumed to interact with a Coulomb interaction $e_{1} e_{2} /|\mathbf{r}|$. To make the problem simple, we shall assume the attainment of local thermal equilibrium.

When the external charges $Q$ and $\bar{Q}$ are introduced into the medium, the medium fermions will re-arrange themselves in both coordinates and momenta to reach a new local thermal equilibrium. The total number of the medium particles in the system is not a constant of motion but is determined by the condition of thermal equilibrium, maintained by the thermal bath.

When the external charges $Q$ and $\bar{Q}$ are separated by a distance $\mathbf{R}$ at a temperature $T$, the self-consistent rearrangement of the medium charged fermion particles leads to a potential $V(\mathbf{r}, \mathbf{R})$ at a point $\mathbf{r}$ and under a local thermal equilibrium, the momentum distribution of the medium particles at $\mathbf{r}$ in the Born-Oppenheimer treatment is 
given by

$$
f_{ \pm}(\mathbf{r}, \mathbf{p}, \mathbf{R})=\frac{1}{\exp \left\{\left[p+e_{ \pm} V(\mathbf{r}, \mathbf{R})\right] / T\right\}+1} .
$$

Here and henceforth, the + and - subscripts designate quantities for the positive and negative medium particles respectively.

From the above Wigner function distribution, we can obtain various thermodynamic quantities. The integration of the Wigner function over all momenta gives the spatial number density distribution $n_{ \pm}(\mathbf{r}, \mathbf{R})$ at $\mathbf{r}$, when $Q$ and $\bar{Q}$ are separated by $\mathbf{R}$,

$$
n_{ \pm}(\mathbf{r}, \mathbf{R})=\frac{g}{2 \pi^{2}} \int p^{2} d p f_{ \pm}(\mathbf{r}, \mathbf{p}, \mathbf{R})
$$

where $g$ is the degeneracy of the levels. We can consider the high temperature case for which it is useful to expand various quantities as a power series of $e_{ \pm} V\left((\mathbf{r}, \mathbf{R}) / T\right.$. Up to the second order in $\left[e_{ \pm} V(\mathbf{r}, \mathbf{R}) / T\right]^{2}$, the medium particle density is

$$
n_{ \pm}(\mathbf{r}, \mathbf{R})=n_{ \pm}^{0}\left\{1-a_{1}\left[e_{ \pm} V(\mathbf{r}, \mathbf{R}) / T\right]+a_{2}\left[e_{ \pm} V(\mathbf{r}, \mathbf{R}) / T\right]^{2}\right\}
$$

where

$$
\begin{gathered}
n_{ \pm}^{0}=\frac{g T^{3}}{2 \pi^{2}} \frac{3}{4} \zeta(3) \Gamma(3), \\
a_{1}=\frac{\frac{1}{2} \zeta(2)}{\frac{3}{4} \zeta(3)}=0.91233
\end{gathered}
$$

and

$$
a_{2}=\frac{\frac{1}{2} \zeta(1)}{\frac{3}{4} \zeta(3)}=0.3845 .
$$

In passing, we note that if the medium particles obeys Boltzmann statistics, the coefficients $a_{1}$ and $a_{2}$ would equal $a_{1}=1$ and $a_{2}=0.5$, as it follows from the expansion of the well-known Boltzmann distribution $n_{ \pm}(\mathbf{r}, \mathbf{R})=$ $n_{ \pm}^{0} \exp \left\{-e_{ \pm} V(\mathbf{r}, \mathbf{R}) / T\right\}$ for Boltzmann particles in an external field. The values of the $a_{1}$ and $a_{2}$ coefficients for Boltzmann statistics differ only slightly from the corresponding values in (7) and (8) for fermion particles.

For the Fermi-Dirac medium particles, the entropy density at $\mathbf{r}$, when $Q$ and $\bar{Q}$ are separated by $\mathbf{R}$, is given by

$$
\sigma_{ \pm}(\mathbf{r}, \mathbf{R})=\frac{g}{2 \pi^{2}} \int p^{2} d p\left\{-f_{ \pm} \ln f_{ \pm}-\left(1-f_{ \pm}\right) \ln \left(1-f_{ \pm}\right)\right\}
$$

Upon substituting the Fermi-Dirac distribution of Eq. (3) into the above equation, we find

$$
\begin{aligned}
\sigma_{ \pm}(\mathbf{r}, \mathbf{R}) & =\frac{g}{2 \pi^{2}} \int p^{2} d p f_{ \pm}(\mathbf{r}, \mathbf{p}, \mathbf{R})\left\{\frac{4 p}{3}+e_{ \pm} V(\mathbf{r}, \mathbf{R})\right\} / T \\
& \equiv\left\langle\frac{4 p}{3}+e_{ \pm} V(\mathbf{r}, \mathbf{R})\right\rangle / T
\end{aligned}
$$

and we obtain

$$
\sigma_{ \pm}(\mathbf{r}, \mathbf{R})=\sigma_{ \pm}^{0}\left\{1-b_{1}\left[e_{ \pm} V((\mathbf{r}, \mathbf{R}) / T]+b_{2}\left[e_{ \pm} V(\mathbf{r}, \mathbf{R}) / T\right]^{2}\right\}\right.
$$

where

$$
\begin{gathered}
\sigma_{ \pm}^{0}=\frac{g T^{3}}{2 \pi^{2}} \frac{7}{8} \zeta(4) \frac{4}{3} \Gamma(4), \\
b_{1}=\frac{\frac{3}{4} \zeta(3)\left[\frac{4}{3} \Gamma(4)-\Gamma(3)\right]}{\frac{7}{8} \zeta(4) \frac{4}{3} \Gamma(4)}=0.7139
\end{gathered}
$$


and

$$
b_{2}=\frac{\frac{1}{2} \zeta(2)\left[\frac{4}{3} \frac{\Gamma(4)}{2}-\Gamma(3)\right]}{\frac{7}{8} \zeta(4) \frac{4}{3} \Gamma(4)}=0.2171 .
$$

Here we have purposely written the numerators of the $b_{1}$ and $b_{2}$ coefficients as a difference where the first term comes from $\langle 4 p / 3\rangle$ and the second term comes from $\left\langle e_{ \pm} V\right\rangle$ of Eq. (10).

The evaluation of various thermodynamical quantities requires the knowledge of $V(\mathbf{r}, \mathbf{R})$. To determine $V(\mathbf{r}, \mathbf{R})$ self-consistently, we have the charge density at the point $\mathbf{r}$, when $Q$ and $\bar{Q}$ are separated by $\mathbf{R}$,

$$
\rho_{\text {total }}(\mathbf{r}, \mathbf{R})=q \delta\left(\mathbf{r}+\frac{\mathbf{R}}{2}\right)-q \delta\left(\mathbf{r}-\frac{\mathbf{R}}{2}\right)+e_{+} n_{+}(\mathbf{r}, \mathbf{R})+e_{-} n_{-}(\mathbf{r}, \mathbf{R}) .
$$

Using the number density distribution given in Eq. (5), the charge density, up to the second power in $e_{ \pm} V(\mathbf{r}, \mathbf{R}) / T$, becomes

$$
\rho_{\text {total }}(\mathbf{r}, \mathbf{R})=q \delta\left(\mathbf{r}+\frac{\mathbf{R}}{2}\right)-q \delta\left(\mathbf{r}-\frac{\mathbf{R}}{2}\right)-n_{0} a_{1} q^{2} V(\mathbf{r}, \mathbf{R}),
$$

where $n_{0}=n_{+}^{0}+n_{-}^{0}$ and the zeroth-order and second-order terms of Eq. (5) cancel out on account of the presumed charge neutrality of the system for which $n_{+}^{0}=n_{-}^{0}$. The Poisson equation for the potential is then given by

$$
\nabla_{\mathbf{r}}^{2} V(\mathbf{r}, \mathbf{R})=-4 \pi\left\{q \delta\left(\mathbf{r}+\frac{\mathbf{R}}{2}\right)-q \delta\left(\mathbf{r}-\frac{\mathbf{R}}{2}\right)-n_{0} a_{1} q^{2} V(\mathbf{r}, \mathbf{R})\right\},
$$

which has the solution

$$
\begin{aligned}
V(\mathbf{r}, \mathbf{R}) & =\frac{q e^{-\mu r_{+}}}{r_{+}}-\frac{q e^{-\mu r_{-}}}{r_{-}}, \\
r_{ \pm} & =|\mathbf{r} \pm \mathbf{R} / 2|, \\
\mu & =\sqrt{\frac{4 \pi n_{0} a_{1} q^{2}}{T}}=\frac{1}{r_{D}},
\end{aligned}
$$

where $\mu$ is the Debye mass and $r_{D}$ is the Debye screening length.

\section{VARIATION OF NUMBER DENSITY AND ENTROPY DENSITY WITH $R$ IN DEBYE SCREENING}

The simple solution $V(\mathbf{r}, \mathbf{R})$ in Eq. (18) of the last section allows us to have a profile of the self-consistent medium particle number density and entropy density in all spatial points at local thermal equilibrium at $T$. In Eqs. (5) and (11), the coefficients of $a_{1}, a_{2}, b_{1}$ and $b_{2}$ are all positive. For positive medium particles, the first-order increment in the number density $n_{+}(\mathbf{r}, \mathbf{R})$ and $\sigma_{+}(\mathbf{r}, \mathbf{R})$, [also $u_{+}(\mathbf{r}, \mathbf{R})$ in Eq. (32)] are therefore measured by $\left[-e_{+} V(\mathbf{r}, \mathbf{R}) / T\right]$ illustrated in Fig. 2 as a function of $\rho / r_{D}$ and $z / r_{D}$. As one observes, the first-order contributions, given by $-e_{+} V(\mathbf{r}, \mathbf{R}) / T$, represent a depletion for the positive medium particles near the positive static charge $Q$ at $-\mathbf{R} / 2$, and an enhancement near the negative static charge $\bar{Q}$ at $\mathbf{R} / 2$. The degree of depletion and the degree of enhancement are equal and opposite to each other. When we sum over all spatial points, the sum of the first-order depletion and enhancement cancel each other to give a zero total contribution.

The second-order contributions are proportional to $\left[e_{+} V(\mathbf{r}, \mathbf{R}) / T\right]^{2}$ and are always positive. They are illustrated in Fig. 3 as a function of $\rho / r_{D}$ and $z / r_{D}$ for different $Q-\bar{Q}$ separations. They always enhance the number density and the entropy density. The enhancement is small when the two static charges are close together in Fig. $3(a)$, as there is a substantial cancellation of the two terms in Eq. (18 a). The enhancement reaches a constant value when the static charges $Q$ and $\bar{Q}$ reaches a separation of 1-2 units of the Debye screening length as shown in Fig. $3 b$ and $3 c$.

If one integrates over all spatial points to obtain the total number of positive charge medium particles, one finds that the integration over the first-order term, $\int d \mathbf{r}\left[-e_{+} V(\mathbf{r}, \mathbf{R}) / T\right]$, is zero because the depletion cancels the enhancement. However, the second-order contributions always give a positive contribution, and the number of positive medium particles, measured relative to its corresponding quantity in the absence of $Q$ and $\bar{Q}$, is given by

$$
N_{+}(R)=\int d \mathbf{r}\left\{n_{+}(\mathbf{r}, \mathbf{R})-n_{+}^{0}\right\}=n_{+}^{0} \int d \mathbf{r} a_{2}\left[e_{+} V(\mathbf{r}, \mathbf{R}) / T\right]^{2} .
$$




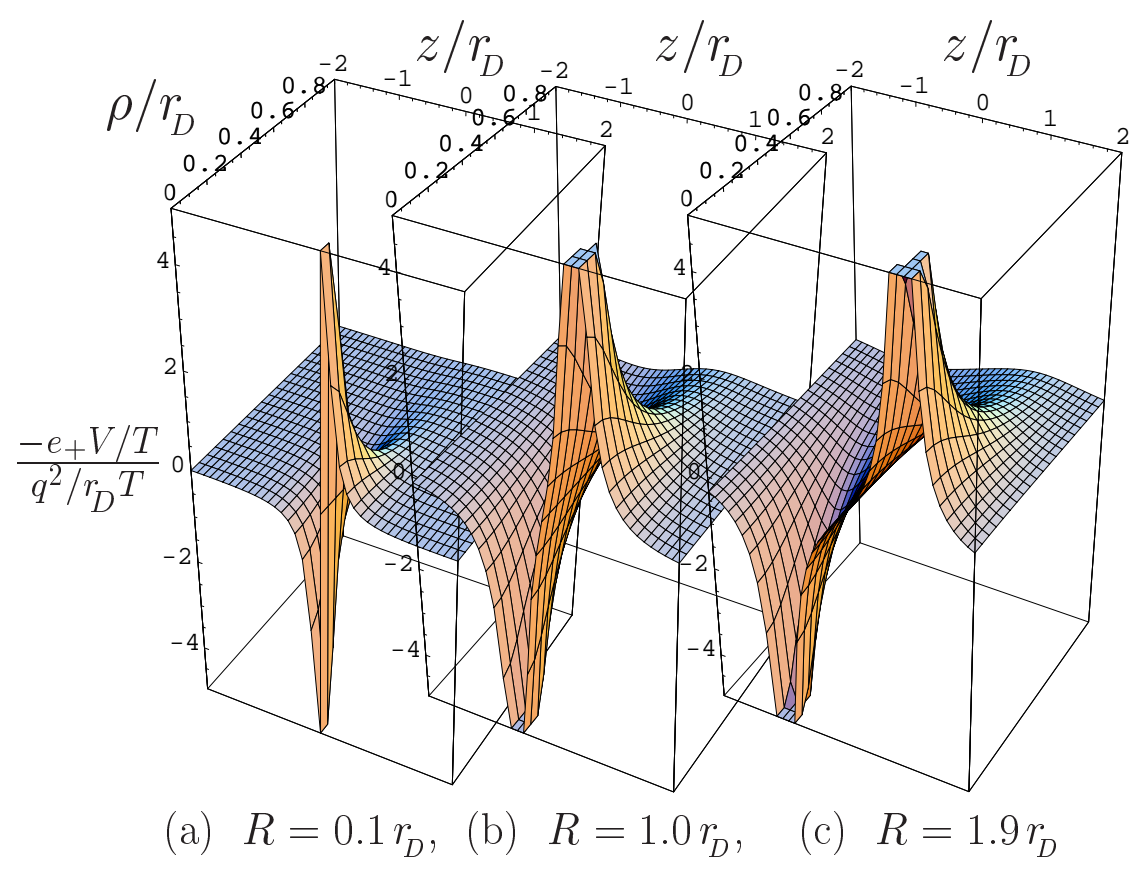

FIG. 2: The first-order term $\left[-e_{+} V(\rho z, T) / T\right]$ in units of $q^{2} / r_{D}$ in Eqs. (5), (11), and (32) that contributes to the increments in $n_{+}(\rho z, R), \sigma_{+}(\rho z, R)$, and $u_{+}(\rho z, R)$, as a function of the spatial coordinates $\rho / r_{D}$ and $z / r_{D}$. Fig. $2(a)$ is for $R=0.1 r_{D}$, Fig. $2(b)$ for $R=1.0 r_{D}$, and Fig. $2(c)$ for $R=1.9 r_{D}$.

We can write the above as

$$
\frac{N_{+}(R)}{N_{+}(R \rightarrow \infty)}=\eta(R)
$$

where

$$
\eta(R)=\frac{1}{4 \pi} \int d \zeta\left[\frac{e^{-\zeta_{+}}}{\zeta_{+}}-\frac{e^{-\zeta_{-}}}{\zeta_{-}}\right]^{2},
$$

with $\boldsymbol{\zeta}=\mathbf{r} / r_{D}, \zeta_{ \pm}=|\mathbf{r} \pm \mathbf{R} / \mathbf{2}| / r_{D}, \eta(0)=0$, and $\eta(R \rightarrow \infty)=1$. In Eq. (20), $N_{+}(R \rightarrow \infty)$ is the increment in the number of positive medium particles when $Q$ and $\bar{Q}$ are far separated,

$$
N_{+}(R \rightarrow \infty)=n_{+}^{0} 3 a_{2}\left(\frac{q^{2}}{r_{D} T}\right)^{2} \frac{4 \pi}{3} r_{D}^{3} .
$$

The increase in the total number of positive medium particles is small when $Q$ and $\bar{Q}$ are close together, and the increase saturates when $R$ reaches a few units of the Debye screening length $r_{D}$. For our charge-neutral system, we obtained from Eq. (5) in a similar way $N_{-}(R)=N_{+}(R)$.

Similarly, the entropy density of the medium particles is depleted near the static charge of the same sign, and is enhanced in the vicinity of the static charge of the opposite sign. When integrated over all spatial points, the depletion and the enhancement cancel to the first order. The second-order contributions always give a positive total entropy,

$$
T S_{ \pm}(R)=T \int d \mathbf{r}\left\{\sigma_{ \pm}(\mathbf{r}, \mathbf{R})-\sigma_{ \pm}^{0}\right\}=T \sigma_{ \pm}^{0} \int d \mathbf{r} b_{2}\left[e_{ \pm} V(\mathbf{r}, \mathbf{R}) / T\right]^{2},
$$

which is small at small $R$ and saturates at large $R$. We can write the above as

$$
\frac{T S_{ \pm}(R)}{T S_{ \pm}(R \rightarrow \infty)}=\eta(R)
$$

where

$$
T S_{ \pm}(R \rightarrow \infty)=T \sigma_{ \pm}^{0} 3 b_{2}\left(\frac{q^{2}}{r_{D} T}\right)^{2} \frac{4 \pi}{3} r_{D}^{3}
$$




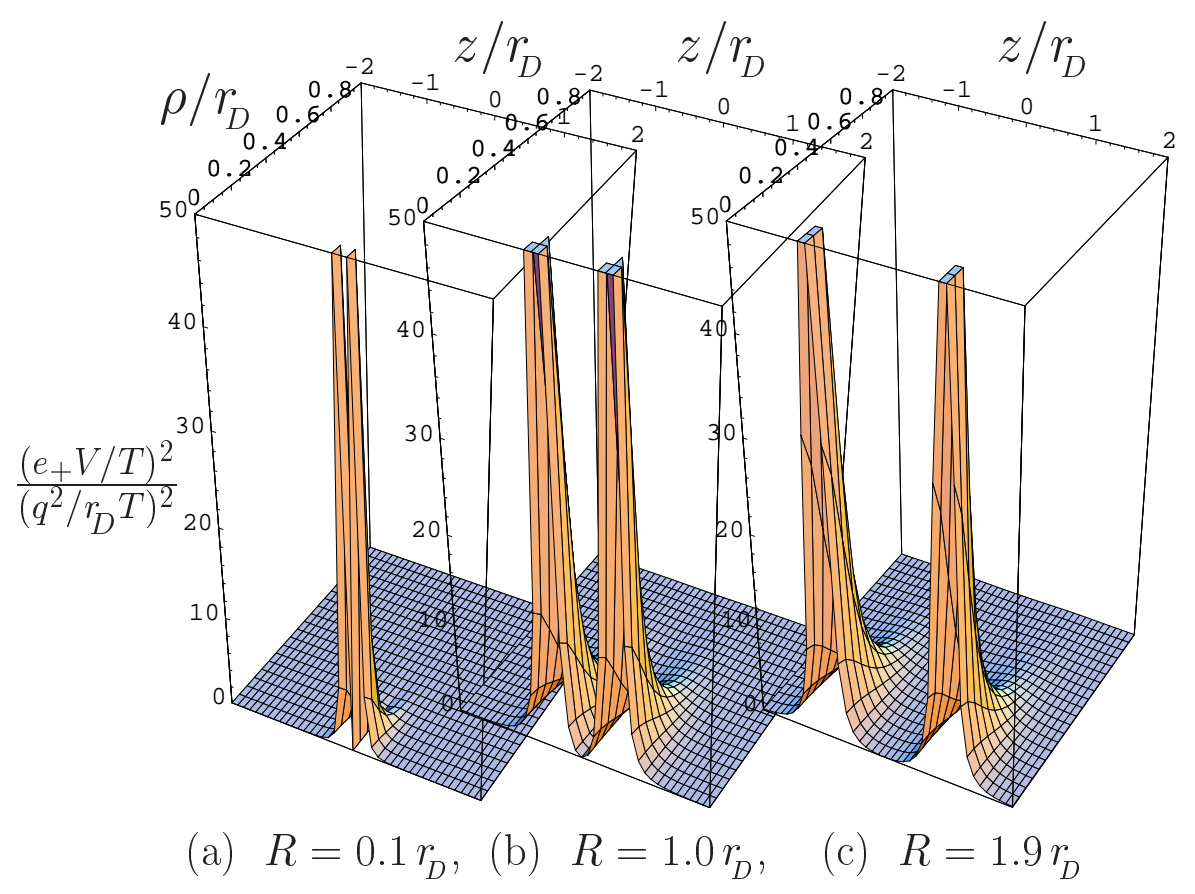

FIG. 3: The second-order term $\left(e_{+} V(\rho z, R) / T\right)^{2}$ in units of $\left(q^{2} / r_{D} T\right)^{2}$ in Eqs. (5), (11), and (32) that contributes to $n_{+}(\rho z, R)$, $\sigma_{+}(\rho z, R)$, and $u_{+}(\rho z, R)$, as a function of the spatial coordinates $\rho / r_{D}$ and $z / r_{D}$. Fig. 3(a) is for $R=0.1 r_{D}$, Fig. 3(b) for $R=1.0 r_{D}$, and Fig. $3(c)$ for $R=1.9 r_{D}$.

A comparison of $N_{ \pm}$and $T S_{ \pm}$shows that

$$
\frac{N_{ \pm}(R)}{N_{ \pm}(R \rightarrow \infty)}=\frac{T S_{ \pm}(R)}{T S_{ \pm}(R \rightarrow \infty)}=\eta(R)
$$

If we define $N(R)=N_{+}+N_{-}(R)$, and $T S(R)=T S_{+}(R)+T S_{-}(R)$, the increment of the total number and entropy of the medium particles due to the presence of $Q$ and $\bar{Q}$ (measured relative to the corresponding quantities in the absence of $Q$ and $\bar{Q}$ ), is

$$
\frac{N(R)}{N(R \rightarrow \infty)}=\frac{T S(R)}{T S(R \rightarrow \infty)}=\eta(R)
$$

Thus, the ratios $T S(R) / T S(R \rightarrow \infty), N_{ \pm}(R) / N_{ \pm}(R \rightarrow \infty)$, and $T S(R) / T S(R \rightarrow \infty)$ [also $U(R) / U(R \rightarrow \infty)$ as we shall see in the next section] behave in the same way as a function of $R$. We show the behavior of $\eta(R)=$ $T S(R) / T S(R \rightarrow \infty)$ for the Debye screening case in Fig $1(b)$ and it has the same shape as $T S_{1}(R)$ obtained in the lattice gauge calculations shown in Fig. 1(a).

One can therefore understand that as the result of constraining the system to be in contact with a thermal bath in the grand canonical ensemble, the medium particle numbers and entropy increase with increasing $R$ to maintain a thermal equilibrium until they saturate at large separation. The thermal bath is therefore a participant in altering the content of the medium particles, when the $Q$ is separating from the antiquark $\bar{Q}$. (See Section VI for a discussion on the role of the thermal bath).

\section{VARIATION OF THE INTERNAL ENERGY WITH $R$ IN DEBYE SCREENING}

Previously in quenched QCD, we find a relationship between the total internal energy and the $Q-\bar{Q}$ potential [27]. As the problem of non-perturbative QCD so intrinsically complicated, a thorough understanding of such a relationship in an analogous, but not identical, problem in QED is worth having. We are therefore motivated to examine the mechanism of Debye screening due to the medium particles in QED. Of particular interest is to see whether the relationship between the total internal energy and the $Q-\bar{Q}$ potential in Debye screening in QED resembles a similar relationship in lattice gauge theory obtained previously in QCD [27]. 
When $Q$ and $\bar{Q}$ separated by a distance $R$ are screened by medium particles, the total internal energy $U_{\text {total }}(R)$ of the system of medium particles, $Q$, and $\bar{Q}$ is the sum of the kinetic energy of the medium particles and the interaction energies of the the medium particles, $Q$, and $\bar{Q}$, when the medium particles have re-arranged themselves self-consistently,

$$
\begin{aligned}
U_{\text {total }}(R) & =\int d \mathbf{r}\left\{\frac{g}{2 \pi^{2}} \int p^{2} d p\left[f_{+}(\mathbf{r}, \mathbf{p}, \mathbf{R})+f_{-}(\mathbf{r}, \mathbf{p}, \mathbf{R})\right] p\right\} \\
& +\frac{1}{2} \int d \mathbf{r}\left\{e_{+} \delta\left(\mathbf{r}+\frac{\mathbf{R}}{2}\right)+e_{-} \delta\left(\mathbf{r}-\frac{\mathbf{R}}{2}\right)+e_{+} n_{+}(\mathbf{r}, \mathbf{R})+e_{-} n_{-}(\mathbf{r}, \mathbf{R})\right\} V(\mathbf{r}, \mathbf{R}) \\
& -\int d \mathbf{r}\left(u_{+}^{0}+u_{-}^{0}\right),
\end{aligned}
$$

where $u_{ \pm}^{0}$ is the internal energy density of the medium in the absence of $Q$ and $\bar{Q}$ given by

$$
u_{ \pm}^{0}=\frac{g T^{4}}{2 \pi^{2}} \frac{7}{8} \zeta(4) \Gamma(4)
$$

and $U_{\text {total }}(R)$ is measured relative to the total internal energy of the system in the absence of $Q$ and $\bar{Q}, U_{\text {medium }}^{0}=$ $\int d \mathbf{r}\left(u_{+}^{0}+u_{-}^{0}\right)$. Using the solution of $V(\mathbf{r}, \mathbf{R})$ in Eq. (18) and excluding the infinite energies of a point source acting on itself, Eq. (28) gives

$$
U_{\text {total }}(R)=-\frac{q^{2} e^{-\mu R}}{R}-\frac{q^{2}}{r_{D}}+\int d \mathbf{r}\left[\left\{u_{+}(\mathbf{r}, \mathbf{R})-u_{+}^{0}\right\}+\left\{u_{-}(\mathbf{r}, \mathbf{R})-u_{-}^{0}\right\}\right],
$$

where $u_{ \pm}(\mathbf{r}, \mathbf{R})$ are the internal energy density of the positive and negative charged medium particles in the presence of $Q$ and $\bar{Q}$ given by

$$
\begin{aligned}
u_{ \pm}(\mathbf{r}, \mathbf{R}) & =\frac{g}{2 \pi^{2}} \int p^{2} d p f_{ \pm}(\mathbf{r}, \mathbf{p}, \mathbf{R})\left\{p+\frac{e_{ \pm} V(\mathbf{r}, \mathbf{R})}{2}\right\} \\
& \equiv\left\langle p+\frac{e_{ \pm} V(\mathbf{r}, \mathbf{R})}{2}\right\rangle .
\end{aligned}
$$

Using the local Fermi-Dirac distribution of Eq. (3), we obtain

$$
u_{ \pm}(\mathbf{r}, \mathbf{R})=u_{ \pm}^{0}\left\{1-c_{1}\left[e_{ \pm} V((\mathbf{r}, \mathbf{R}) / T]+c_{2}\left[e_{ \pm} V(\mathbf{r}, \mathbf{R}) / T\right]^{2}\right\},\right.
$$

where $c_{1}$ and $c_{2}$ are positive constants,

$$
c_{1}=\frac{\frac{3}{4} \zeta(3)\left[\Gamma(4)-\frac{\Gamma(3)}{2}\right]}{\frac{7}{8} \zeta(4) \Gamma(4)}=0.7933
$$

and

$$
c_{2}=\frac{\frac{1}{2} \zeta(2)\left[\frac{\Gamma(4)}{2}-\frac{\Gamma(3)}{2}\right]}{\frac{7}{8} \zeta(4)}=0.2895 .
$$

A comparison of $u_{ \pm}(\mathbf{r}, \mathbf{R})$ in Eq. (32) with $n_{ \pm}(\mathbf{r}, \mathbf{R})$ and $u_{ \pm}(\mathbf{r}, \mathbf{R})$ in Eqs. (5) and (11) indicates that the first-order and second-order contributions to $u_{ \pm}(\mathbf{r}, \mathbf{R})$ behave in the same way as those of $n_{ \pm}(\mathbf{r}, \mathbf{R})$ and $\sigma_{ \pm}(\mathbf{r}, \mathbf{R})$. The first-order increment of the internal energy density of the medium particles is suppressed near the static charge of the same sign, and is enhanced near the static charge of the opposite sign as shown in Fig. 2, while the second-order contributions to the internal energy are always positive as shown in Fig. 3. The first-order contributions cancel each other, when they are integrated over all the spatial points. However, the second-order contributions are always positive, and the integration of the second-order contributions always yield a positive quantity. The total medium internal energy of the positive and negative medium particles, measured relative to the corresponding quantities in the absence of $Q$ and $\bar{Q}$, are

$$
U_{ \pm}(R)=\int d \mathbf{r}\left\{u_{ \pm}(\mathbf{r}, \mathbf{R})-u_{ \pm}^{0}\right\}=n_{ \pm}^{0} \int d \mathbf{r} c_{2}\left[e_{ \pm} V(\mathbf{r}, \mathbf{R}) / T\right]^{2}
$$


Relative to the total internal energy in the absence of $Q$ and $\bar{Q}$, the total internal energy in the presence of $Q$ and $\bar{Q}$, is

$$
U_{\text {medium }}(R)-U_{\text {medium }}^{0}=U_{+}(R)+U_{-}(R) .
$$

Eq. (30) can therefore be written as

$$
U_{\text {total }}(R)=-\frac{q^{2} e^{-\mu R}}{R}-\frac{q^{2}}{r_{D}}+U_{\text {medium }}(R)-U_{\text {medium }}^{0}
$$

From Eqs. (35), (19), and (21), the quantity $U_{ \pm}(R)$ can be written as

$$
\frac{U_{ \pm}(R)}{U_{ \pm}(R \rightarrow \infty)}=\eta(R)
$$

where

$$
U_{ \pm}(R \rightarrow \infty)=u_{ \pm}^{0} 3 c_{2}\left(\frac{q^{2}}{r_{D} T}\right)^{2} \frac{4 \pi}{3} r_{D}^{3}
$$

A comparison of $N_{ \pm}$and $T S_{ \pm}$shows that

$$
\frac{U_{ \pm}(R)}{U_{ \pm}(R \rightarrow \infty)}=\frac{N_{ \pm}(R)}{N_{ \pm}(R \rightarrow \infty)}=\frac{T S_{ \pm}(R)}{T S_{ \pm}(R \rightarrow \infty)}=\eta(R) .
$$

Consequently, if we define $U(R)=U_{+}(R)+U_{-}(R)$, we also have

$$
\frac{U(R)}{U(R \rightarrow \infty)}=\frac{N(R)}{N(R \rightarrow \infty)}=\frac{T S(R)}{T S(R \rightarrow \infty)}=\eta(R) .
$$

In this simple model of Debye screening, the ratios of $T S(R) / T S(R \rightarrow \infty), N(R) / N(R \rightarrow \infty)$ and $U(R) / U(R \rightarrow \infty)$ are equal and their behavior is shown in Fig. 1(b). The entropy, total number, and internal energy of the medium particles (relative to the corresponding quantities in the absence of $Q$ and $\bar{Q}$ ) is zero at $R=0$ and increases as a function of $R$ until they saturate when the separation $R$ reaches a few units of the Debye screening length.

\section{SCHRÖDINGER EQUATION FOR $Q-\bar{Q}$ SYSTEM IN DEBYE SCREENING}

As the dynamics of a quark and an antiquark in a quark-gluon plasma is very complicated, it is worth having a good understanding of the mechanism of screening and its effects on the interaction between a $Q$ and an antiquark $\bar{Q}$ in an analogous, but not identical, problem. We are therefore motivated to examine the mechanism of Debye screening due to the medium particles in QED. Of particular interest is to obtain the relationship between the $Q-\bar{Q}$ potential in the Hamiltonian and the total internal energy $U_{\text {total }}$, in order to find out whether this relationship resembles a similar relationship in lattice gauge theory obtained previously in Ref. 27].

It is however somewhat tricky to determine the Hamiltonian for the $Q$ and $\bar{Q}$ system in a medium under Debye screening (and analogously, but not identically, under color-charge screening in QCD). We can follow the basic principles of statistical physics as described by Landau and Lifshitz [53]. Accordingly, we start with a closed system of a heavy $Q$ and $\bar{Q}$ with medium particles and consider a small "subsystem" $S$ that contains the $Q$, the $\bar{Q}$, and the medium. As the number of medium particles of the whole closed system is very large, the number of medium particles contained in the small subsystem $S$ can still be very large, and a statistical description of this small subsystem $S$ is applicable. This subsystem $S$ is not a closed system and it undergoes all kinds of interaction and medium particle exchanges with the complementary part $S^{\prime}$ of the whole system. We can describe this subsystem $S$ to be in contact with a very large complementary part $S^{\prime}$, which we can call a "thermal bath" in this connection. The QED system discussed in the last few sections or the QCD system in lattice gauge calculations, (the so-called "system" of $Q, \bar{Q}$, and medium particles in contact with a thermal bath), corresponds in actual fact to the "subsystem" $S$ out of the whole closed system $S+S^{\prime}$.

Under a perturbation of the subsystem $S$ away from thermal equilibrium such as occurs in the displacement of $Q$ relative to $\bar{Q}$, the medium in the subsystem $S$ will respond to the perturbation and will relax to a new state of thermal equilibrium after a certain relaxation time, $t_{\text {relax }}(S)$. For example, from the results concerning the medium entropy and number contents in the subsystem $S$ as a function of the separation between $Q$ and $\bar{Q}$ obtained in the last few 
sections (Fig. 1), we know that under a displacement of the relative separation of the $Q$ and $\bar{Q}$, the medium particles will exchange between $S$ and the thermal bath $S^{\prime}$ in order to make the subsystem $S$ under thermal equilibrium. In this case, the relaxation time $t_{\text {relax }}(S)$ corresponds to the exchange of medium particles through imaginary boundaries between $S$ and the thermal bath $S^{\prime}$. Relaxation time grows smaller as the subsystem $S$ decreases in size [53]. For a small subsystem $S$, this relaxation time $t_{\text {relax }}(S)$ can be very short. On the other hand, for heavy $Q$ and $\bar{Q}$ in the subsystem, the period of $Q-\bar{Q}$ relative motion, $t_{Q \bar{Q}}$, can be relatively long because of the large mass of $Q$ and $\bar{Q}$. The period $t_{Q \bar{Q}}$ can be so much greater than the medium relaxation time $t_{\text {relax }}(S), t_{Q \bar{Q}}>>t_{\text {relax }}(S)$, that the medium can be approximately considered as reaching a state of thermal equilibrium approximately instantaneously, at any time during the (supposedly slow) motion of the heavy quark $Q$ and antiquark $\bar{Q}$. For the medium particles, this is just the Born-Oppenheimer approximation for the description of the states of the medium particles in the subsystem $S$, as presented in the last few sections and used in lattice gauge calculations to obtain the medium particle configurations in QCD.

In what sense can energy and entropy be considered conserved under a periodic motion of the $Q$ and $\bar{Q}$ in the medium? The whole closed system consists of the subsystem $S^{\prime}$ and $S$, and the subsystem $S$ is not a closed system. From the results of the last few sections, we know that if we move the $Q$ closer relative to the $\bar{Q}$ in the subsystem $S$, then the medium particles (and its entropy and energy contents) will move from the subsystem $S$ into the complementary part $S^{\prime}$ so as to maintain thermal equilibrium in the subsystem $S$. When we move the $Q$ farther relative to the $\bar{Q}$, then the medium particles, entropy, and energy contents will move from the complementary system $S^{\prime}$ back into the subsystem $S$ so as to maintain thermal equilibrium in the subsystem $S$. For slow periodic motion of $Q$ and $\bar{Q}$, the motion can be so slow that the exchange of medium particles between $S$ and $S^{\prime}$ can be approximated as taking place with no excitation of the medium. In this sense, the idealized periodic motion of $Q$ and $\bar{Q}$ can thus be adiabatic in the lowest order with an "adiabatic" exchanging the energy content and the entropy content of the medium particles back and forth between the subsystem $S$ and the complementary system $S^{\prime}$. Additional interactions of the bound periodic $Q-\bar{Q}$ states with the medium particles that lead to the non-adiabatic excitation of both objects can then be considered in higher-order approximations.

Thus, in this adiabatic picture of $t_{Q \bar{Q}}>>t_{\text {relax }}(S)$, the $Q$ and $\bar{Q}$ experience the interactions from all medium particles which adjusts themselves (within a relaxation time which is taken to be so small as to be approximately instantaneous) at all instances of the dynamical motion of the $Q$ and $\bar{Q}$. The potential energy of the $Q$ and $\bar{Q}$ at a separation $r$ in the Hamiltonian for the $Q$ and $\bar{Q}$ is half of the integral of the product of the local point charges of the $Q$ and $\bar{Q}$ with their local potentials $V(\mathbf{r}, \mathbf{R})$ arising from a self-consistent rearrangement of all particles when $Q$ and $\bar{Q}$ are at the separation $\mathbf{R}$, excluding the infinite self-energy contributions. In addition to the potential energy of the $Q$ and $\bar{Q}$, the Hamiltonian for the $Q-\bar{Q}$ system consists also of the kinetic energy of $Q$ and $\bar{Q}$. The Hamiltonian for the $Q-\bar{Q}$ system is therefore given by

$$
\begin{aligned}
H & =\frac{\mathbf{p}_{Q}^{2}}{2 m_{Q}}+\frac{\mathbf{p}_{\bar{Q}}^{2}}{2 m_{\bar{Q}}}+\frac{1}{2} \int d \mathbf{r}\left[e_{+} \delta\left(\mathbf{r}+\frac{\mathbf{R}}{2}\right)+e_{-} \delta\left(\mathbf{r}-\frac{\mathbf{R}}{2}\right)\right] V(\mathbf{r}, \mathbf{R}) \\
& =\frac{\mathbf{p}_{Q}^{2}}{2 m_{Q}}+\frac{\mathbf{p}_{\bar{Q}}^{2}}{2 m_{\bar{Q}}}+\frac{1}{2}\left[\left.e_{+} V(\mathbf{r}, \mathbf{R})\right|_{\mathbf{r}=-\mathbf{R} / 2}+\left.e_{-} V(\mathbf{r}, \mathbf{R})\right|_{\mathbf{r}=\mathbf{R} / 2}\right] .
\end{aligned}
$$

Upon making the change of the variables from $\mathbf{p}_{Q}$ and $\mathbf{p}_{\bar{Q}}$ to the center-of-mass momentum $\mathbf{P}_{\mathrm{CM}}=\mathbf{p}_{Q}+\mathbf{p}_{\bar{Q}}$ and the relative momentum $\mathbf{p}_{R}=\left(\mathbf{p}_{Q}-\mathbf{p}_{\bar{Q}}\right) / 2$, and using the solution $V(\mathbf{r}, \mathbf{R})$ of Eq. (18), we find from the above equation

$$
H=\frac{\mathbf{P}_{\mathrm{CM}}^{2}}{2\left(m_{Q}+m_{\bar{Q}}\right)}+\frac{\mathbf{p}_{R}^{2}}{2 \mu_{\mathrm{red}}}-\frac{q^{2} e^{-\mu R}}{R}-\frac{q^{2}}{r_{D}},
$$

where $\mu_{\text {red }}=m_{Q} m_{\bar{Q}} /\left(m_{Q}+m_{\bar{Q}}\right)$ is the reduced mass. The Hamiltonian then separates into $H=H_{\mathrm{CM}}+H_{R}$ where $H_{\mathrm{CM}}$ is the Hamiltonian for the free motion of the composite two-body system and $H_{R}$ is the Hamiltonian for the relative motion of $Q$ and $\bar{Q}$,

$$
H_{R}=\frac{\mathbf{p}_{R}^{2}}{2 \mu_{\text {red }}}-\frac{q^{2} e^{-\mu R}}{R}-\frac{q^{2}}{r_{D}} \equiv \frac{\mathbf{p}_{R}^{2}}{2 \mu_{\text {red }}}+U_{Q \bar{Q}}(R)
$$

From the above equation, we recognize that the potential for the $Q-\bar{Q}$ system under screening by the medium, $U_{Q \bar{Q}}(R)$, is given by

$$
U_{Q \bar{Q}}(R)=-\frac{q^{2} e^{-\mu R}}{R}-\frac{q^{2}}{r_{D}} .
$$

The $Q \bar{Q}$ potential $U_{Q \bar{Q}}(R)$ is just the Debye screening potential plus an $R$-independent constant term. 
Based on the "adiabatic" picture of the motion of $Q$ and $\bar{Q}$, the Hamiltonian formulated in Eq. (42) indeed gives correctly the Hamiltonian with the Debye screening potential. The $R$-independent term $-q^{2} / r_{D}$ is also an important part of the screening contribution. We note that if we expand the $Q-\bar{Q}$ Hamiltonian for the case of small $\mu$ representing the screening effects, then Eq. (44) becomes,

$$
H_{R} \sim \frac{\mathbf{p}_{R}^{2}}{2 \mu_{\mathrm{red}}}-\frac{q^{2}(1-\mu R)}{R}-\frac{q^{2}}{r_{D}}=\frac{\mathbf{p}_{R}^{2}}{2 \mu_{\mathrm{red}}}-\frac{q^{2}}{R},
$$

which is the same Hamiltonian as that of the unscreened case. Thus, we reach the interesting result that in the lowest order of the screening parameter $\mu=1 / r_{D}$, a properly calibrated Hamiltonian of a system under screening, with the shift of the level of the potential, $-q^{2} / r_{D}$, is the same Hamiltonian without screening. In practical terms, if we calculate the mass of a $Q-\bar{Q}$ system without screening, we expect that within the lowest order of the screening parameter $\mu$, the mass eigenvalue of the system to be nearly unchanged when screening is present. Numerical calculations of the mass of bound $L=0$ charmonium using the potential model of Ref. [27] indeed shows that the absolute value of the charmonium $L=0$ mass changes only very slightly as a function of temperature up to $1.5 T_{c}$ [30].

From the above discussions in the simple case of Debye screening, we observe that the potential between $Q$ and $\bar{Q}$, $U_{Q \bar{Q}}(R)$, differs from the total internal energy $U_{\text {total }}(R)$. Because of Eqs. (45) and (37), they are related by

$$
U_{Q \bar{Q}}(R)=U_{\text {total }}(R)-\left[U_{\text {medium }}(R)-U_{\text {medium }}^{0}\right] .
$$

It is therefore necessary to subtract out the change of the medium internal energies $\left[U_{\text {medium }}(R)-U_{\text {medium }}^{0}\right]$ from the total internal energy $U_{\text {total }}(R)$ to obtain the $Q-\bar{Q}$ potential $U_{Q \bar{Q}}(R)$ in the grand canonical ensemble. This conclusion for Debye screening supports a similar conclusions in the analogous lattice gauge theory, where we have proved in Eq. (11) of Ref. [27],

$$
U_{Q \bar{Q}}^{(1)}(R, T)=U_{1}(R, T)-\left[U_{g}^{(1)}(R, T)-U_{g 0}(T)\right] .
$$

In the above equation, the superscript (1) refers to the color-singlet state of $Q$ and $\bar{Q}$, and $\left[U_{g}^{(1)}(R, T)-U_{g 0}(T)\right]$ is the increment of gluon energy due to the presence of $Q$ and $\bar{Q}$.

From the above analysis, we conclude that the relationship of Eq. (48) in Ref. 27] is a rather general result for heavy particles under screening in the grand canonical ensemble.

We can understand the results of Eq. (48) [or similarly (47)] from another viewpoint. In a standard description of a $(Q \bar{Q})$ in a medium, we simplify the dynamics by considering first the $(Q \bar{Q})$ states and the deconfined medium states separately as independent unperturbed states. We then include their mutual excitations as perturbative couplings. Thus, in the lowest-order description without perturbative couplings, the $(Q \bar{Q})$ states should be obtained without the excitation of the medium states of deconfined real gluons and vice versa. In the quenched approximation, the deconfined real gluons in the quark-gluon plasma in the Feynman diagram language are those represented by lines with external legs. The change of the medium internal energies, $\left[U_{g}^{(1)}(R, T)-U_{g 0}(T)\right]$ in Eq. (48), represents the excitation of the internal energy states of the deconfined real gluon medium when the separation between the $Q$ and the $\bar{Q}$ changes in the grand canonical ensemble. As the unperturbed states of the $Q-\bar{Q}$ relative motion should be calculated without the excitation of the medium states of deconfined real gluons, we therefore need to subtract the change of the real gluon internal energy from the total internal energy in Eq. (48) to obtain the $Q-\bar{Q}$ potential $U_{Q \bar{Q}}^{(1)}(R, T)$, when the separation between the $Q$ and the $\bar{Q}$ changes.

In the quenched approximation, the subtraction of this change in the internal energy of real gluons as a function of $R$ does not mean that both the real and the virtual gluon degrees of freedom are frozen. Only the real gluon excitation energy degrees of freedom are frozen when we calculate the unperturbed $Q-\bar{Q}$ bound states of relative motion, for reasons we have just given. On the other hand, virtual gluons, which in the Feynman diagram language are represented by gluon lines with the two end points of each line joining onto other quarks and gluons, change their configurations as the separation $R$ between the $Q$ and the $\bar{Q}$ changes. These virtual gluons mediate the interaction between the $Q$ and the $\bar{Q}$. The changes in the virtual gluon configurations modify the interaction between the $Q$ and the $\bar{Q}$, resulting in the screening of the $Q-\bar{Q}$ potential. This type of virtual gluon excitation is not frozen and is included in the calculation of the $Q-\bar{Q}$ potential and the evaluation of bound states of the $Q-\bar{Q}$ relative motion.

The reconfiguration of these virtual gluons can take place in either an adiabatic manner or in the opposite "diabatic" manner depending on whether the time scale of the relaxation of these virtual gluons is short compared to the time scale of the period of the $Q-\bar{Q}$ relative motion. As we explained earlier, the adiabatic description is appropriate for very heavy quark pairs when the relative motion of the $Q$ and the $\bar{Q}$ is slow. This is indeed confirmed by the successful identification of the lattice free energy for a static $Q-\bar{Q}$ pair as the heavy quark $Q-\bar{Q}$ potential, $U_{Q \bar{Q}}^{(1)}(R)$ at $T=0$ [Bali et al. Phys. Rev. D56 2566 (1997)]. For this case of $T=0$, there are no free gluons nor energy excitations of 
free gluon medium states when the separation $R$ between the $Q$ and the $\bar{Q}$ changes, and the total free energy of the system is equal to the total internal energy of the system. The real gluon energy degrees of freedom are absent and frozen but the virtual gluon degrees of freedom adjust themselves as $R$ changes.

The question whether an adiabatic or a diabatic picture is a more appropriate description for the potential arises also in the NN and meson-meson problems at $T=0$ in lattice gauge theory. However aside from the question of adiabaticity, the $Q-\bar{Q}, \mathrm{NN}$, and meson-meson potentials differ in their different degrees of freedom and the methods of calculations. The $Q-\bar{Q}$ potential studied here is a (two-body)-plus-(deconfined medium) problem, while the NN and meson-meson potentials at $T=0$ are (six-body)-plus-(virtual gluons) and (four-body)-plus-(virtual gluons) problems respectively. At $T=0$, a wave function treatment of the lattice gauge correlator results in the correct repulsive potential for the NN potential at short distances [55], while an "adiabatic" potential treatment without using the lattice wave function gives flat NN and meson-meson potentials [56]. On the contrary, however, another "adiabatic" lattice gauge meson-meson potential calculation at $T=0$ gives repulsive and attractive inner cores when different internal degrees of freedom of the light quarks are taken into account [57]. Furthermore, the lattice gauge wave function method of [55] may need additional justifications as questions have been raised in Appendix A of [57] concerning its lattice wave function assumption. While the work of [55] appears to give a correct description, much work remains to be carried out to sort out the differences of the lattice gauge calculations of [55], [56], and [57]. It remains another separate additional question how one can obtain definitive conclusions on the adiabaticity or diabaticity of the (twobody)-plus-(deconfined medium) potential at $T>T_{c}$ from these (six-body)- and (four-body)-plus-(virtual gluon) problems at $T=0$. As many unanswered questions remains to be resolved, the results of [55] cannot yet be used, for the present time at least, to draw conclusions on the adiabaticity or diabaticity of the $Q-\bar{Q}$ potential examined here. Nevertheless, the exploration of the relationship between adiabaticity and the shape of the relative wave function is an interesting subject for future investigations.

\section{AN APPROXIMATE METHOD TO SEPARATE OUT THE $Q-\bar{Q}$ POTENTIAL FROM $U_{1}$}

From the simple model of Debye screening, we observe that up to the first order of $\mathrm{eV} / T$, the internal energy of the medium does not change, but up to the second order the internal energy increases with an increasing separation between $Q$ and $\bar{Q}$. This increase arises from the fact that the thermal equilibrium attained through the contact with a thermal bath in a grand canonical ensemble constrains the occupation numbers of the medium particles, and this newly re-arranged distribution leads to an increase in the number, the entropy, and the internal energy of the medium, as a function of increasing $R$.

Returning now to QCD lattice gauge calculations and noting its similarities with Debye screening of Coulomb charges, we should therefore expect that the number, the entropy, and the internal energy of the gluon medium should likewise increase as function of increasing $R$ between $Q$ and $\bar{Q}$. Indeed, as shown for lattice gauge calculations at a fixed temperature in Fig. 1(a), there is an increase in the entropy of the system as $R$ increases, similar to the analogous Debye screening case shown in Fig. 1(b).

Having understood the behavior of various thermodynamic quantities, we wish to extract the $Q-\bar{Q}$ potential from lattice gauge results. The most reliable way is to carry out additional lattice gauge calculations to obtain $U_{g}^{(1)}(R)$ and $U_{g 0}$. The $Q-\bar{Q}$ potential is then the difference of $U_{1}(R)$ and $U_{g}^{(1)}(R)-U_{g 0}$, as given by Eq. (48). As $U_{g}^{(1)}(R)$ and $U_{g 0}$ in lattice gauge calculations are not yet available, we will try to use another piece of lattice gauge data to obtain the $Q-\bar{Q}$ potential, as least approximately.

We note that in the Debye screening case $U_{\text {medium }}(R)-U_{\text {medium }}^{0}$ is proportional to $T S(R)$, and in the lattice gauge calculations the quantity $T S_{1}(R, T)$ has been calculated. We can look for a similar relationship between the gluon internal energy and the gluon entropy for the quark-gluon plasma. If we succeed in relating $U_{g}^{(1)}(R, T)-U_{g 0}$ to $T S_{1}(R, T)$, then the $Q-\bar{Q}$ potential, $U_{Q \bar{Q}}^{(1)}$, can be determined from $U_{1}(R, T)$ by subtraction using Eq. (48).

The subtraction can be carried out by noting that locally the quark-gluon plasma internal energy density $\epsilon$ is related to its pressure $p$ and entropy density $\sigma$ by the First Law of Thermodynamics,

$$
\epsilon=T \sigma-p
$$

and the quark-gluon plasma pressure $p$ is also related to the plasma energy density $\epsilon$ by the equation of state $p(\epsilon)$ that is presumed known by another lattice gauge calculation. Thus, by expressing $p$ as $(3 p / \epsilon)(\epsilon / 3)$ with the ratio $a(T)=3 p / \epsilon$ given by the known equation of state, the plasma internal energy density $\epsilon$ is related to the entropy density $T \sigma$ by

$$
\epsilon=\frac{3}{3+a(T)} T \sigma .
$$


This is just

$$
\frac{d U_{g}^{(1)}}{d V}=\frac{3}{3+a(T)} \frac{d}{d V} \int d \mathbf{r} T\left(\sigma-\sigma_{0}+\sigma_{0}\right)
$$

where $\sigma_{0}$ is the entropy density in the absence of $Q$ and $\bar{Q}$. Noting that the entropy of the medium for the color-singlet $Q-\bar{Q}$ pair is $T S_{1}=\int d \mathbf{r} T\left(\sigma-\sigma_{0}\right)$ and $U_{g 0}$ is related to $\int d \mathbf{r} T \sigma_{0}$, the above equation leads to

$$
\frac{d\left[U_{g}^{(1)}(R, T)-U_{g 0}(T)\right]}{d V}=\frac{3}{3+a(T)} \frac{T d S_{1}(R, T)}{d V}
$$

and the plasma internal energy integrated over the volume is given by

$$
U_{g}^{(1)}(R, T)-U_{g 0}(T)=\frac{3}{3+a(T)} T S_{1}(R, T) .
$$

But $T S_{1}(R, T)$ has already been obtained as $U_{1}(R, T)-F_{1}(R, T)$. The plasma internal energy is therefore equal to

$$
U_{g}^{(1)}(R, T)-U_{g 0}=\frac{3}{3+a(T)}\left[U_{1}(R, T)-F_{1}(R, T)\right] .
$$

The $Q-\bar{Q}$ potential, $U_{Q \bar{Q}}^{(1)}$, as determined by subtracting the above plasma internal energy from $U_{1}$, is then a linear combination of $F_{1}$ and $U_{1}$ given by [27],

$$
W_{1}(R, T) \equiv U_{Q \bar{Q}}^{(1)}(R, T)=\frac{3}{3+a(T)} F_{1}(R, T)+\frac{a(T)}{3+a(T)} U_{1}(R, T),
$$

where for brevity of notation we have renamed $U_{Q \bar{Q}}^{(1)}(R, T)$ as $W_{1}(R, T)$ and we can define the coefficient of $F_{1}$, $f_{F}=3 /(3+a(T))$, as the $F_{1}$ fraction, and the coefficient of $U_{1}, f_{U}=a(T) /(3+a(T))$, as the $U_{1}$ fraction. The potential $U_{Q \bar{Q}}^{(1)}$ is approximately $F_{1}$ near $T_{c}$ and is approximately $3 F_{1} / 4+U_{1} / 4$ for $T>1.5 T_{c}$ [27].

\section{COMPARISON OF DIFFERENT $Q-\bar{Q}$ POTENTIALS}

In the spectral function analyses, the widths of many color-singlet heavy quarkonium states broaden suddenly at various temperatures [21, 22, 35]. In the most precise calculations for $J / \psi$ using up to 128 time-like lattice slices, the spectrum has a sharp peak for $0.78 T_{c} \leq T \leq 1.62 T_{c}$ and a broad structure with no sharp peak for $1.70 T_{c} \leq T \leq 2.33 T_{c}$ [21]. The spectral peak at the bound state has the same structure and shape at $0.78 T_{c}$ as it is at $1.62 T_{c}$. If one can infer that $J / \psi$ is stable and bound at $0.78 T_{c}$, then it would be reasonable to infer that $J / \psi$ is also bound and stable at $1.62 T_{c}$. The spectral function at $1.70 T_{c}$ has the same structure and shape as the spectral function at $2.33 T_{c}$. If one can infer that $J / \psi$ is unbound at $2.33 T_{c}$, then it would be reasonable to infer that $J / \psi$ become already unbound at $1.70 T_{c}$. We can define the spontaneous dissociation temperature of a quarkonium as the temperature at which the quarkonium changes from bound to unbound and dissociates spontaneously. Thus, from the shape of the spectral functions, the temperature at which the width of a $J / \psi$ quarkonium broadens suddenly from $1.62 T_{c}$ to $1.70 T_{c}$ corresponds to the $J / \psi$ spontaneous dissociation temperature. Spontaneous dissociation temperatures for $\chi_{c}$ and $\chi_{b}$ have been obtained in [22, 35]. We list the heavy quarkonium spontaneous dissociation temperatures obtained from spectral analyses in quenched QCD in Table I. They can be used to test the potential models of $W_{1}\left(\equiv U_{Q \bar{Q}}^{(1)}\right), F_{1}$, and $U_{1}$.

Table I. Spontaneous dissociation temperatures obtained from different analyses.

\begin{tabular}{|c|c|c|c|c|c|c|c|}
\hline & \multicolumn{4}{|c|}{ Quenched QCD } & \multicolumn{3}{c|}{ Full QCD (2 flavors) } \\
\hline States & Spectral Analyses & $W_{1}$ & $F_{1}$ & $U_{1}$ & $W_{1}$ & $F_{1}$ & $U_{1}$ \\
\hline$J / \psi, \eta_{c}$ & $1.62-1.70 T_{c}^{\dagger}$ & $1.62 T_{c}$ & $1.40 T_{c}$ & $2.60 T_{c}$ & $1.42 T_{c}$ & $1.21 T_{c}$ & $2.22 T_{c}$ \\
\hline$\chi_{c}$ & below $1.1 T_{c}^{\natural}$ & unbound & unbound & $1.18 T_{c}$ & $1.05 T_{c}$ & unbound & $1.17 T_{c}$ \\
\hline$\psi^{\prime}, \eta_{c}^{\prime}$ & & unbound & unbound & $1.23 T_{c}$ & unbound & unbound & $1.11 T_{c}$ \\
\hline$\Upsilon, \eta_{b}$ & & $4.1 T_{c}$ & $3.5 T_{c}$ & $\sim 5.0 T_{c}$ & $3.40 T_{c}$ & $2.90 T_{c}$ & $4.18 T_{c}$ \\
\hline$\chi_{b}$ & $1.15-1.54 T_{c}^{\sharp}$ & $1.18 T_{c}$ & $1.10 T_{c}$ & $1.73 T_{c}$ & $1.22 T_{c}$ & $1.07 T_{c}$ & $1.61 T_{c}$ \\
\hline$\Upsilon^{\prime}, \eta_{b}^{\prime}$ & & $1.38 T_{c}$ & $1.19 T_{c}$ & $2.28 T_{c}$ & $1.18 T_{c}$ & $1.06 T_{c}$ & $1.47 T_{c}$ \\
\hline
\end{tabular}

${ }^{\dagger} \operatorname{Ref} .[21], \quad{ }^{\natural} \operatorname{Ref} .[22], \quad{ }^{\sharp}$ Ref.[35]

To evaluate the $Q-\bar{Q}$ potentials in quenched $\mathrm{QCD}$, we use the free energy $F_{1}$ and the internal energy $U_{1}$ obtained by 
Kaczmarek et al. [33] where $F_{1}$ and $U_{1}$ can be parametrized in terms of a screened Coulomb potential with parameters shown in Figs. 2 and 3 of Ref. [27]. For the ratio $a(T)$ from the plasma equation of state in Eq. (55), we use the quenched equation of state of Boyd et al. [58] for quenched QCD. The quantity $a(T)=3 p / \epsilon$ and the $U_{1}$ and $F_{1}$ fractions as a function of $T$ are shown Fig. 1(b) and Fig. 1(c) of Ref. [27] respectively. These quantities allows the specification of the $W_{1} \equiv U_{Q \bar{Q}}^{(1)}$ potential as a function of temperature.

Using quark masses $m_{c}=1.41 \mathrm{GeV}$ and $m_{b}=4.3 \mathrm{GeV}$, we can calculate the binding energies of heavy quarkonia and their spontaneous dissociation temperatures using different potentials in quenched QCD. As a function of temperature, the binding energies and wave functions of charmonia have been presented in Fig. 6 and 7 of Ref. [27] respectively. The bounding energies of bottomia have been presented in Figs. 8 and 9, and the wave functions in Fig. 10 of Ref. [27]. We show the root-mean-square $Q-\bar{Q}$ separation $\sqrt{\left\langle R^{2}\right\rangle}$ of $L=0$ charmonium calculated with $m_{c}=1.41 \mathrm{GeV}$ in the $W_{1}(R)$ potential in quenched QCD as the solid curve in Fig. 4. As one observes, the root-mean-squared separation $R_{\mathrm{RMS}}$ is about $1 \mathrm{fm}$ for $T \sim T_{c}$ and it increases to about $4 \mathrm{fm}$ at $T=1.6 T_{c}$ before it becomes unbound. The large separation is expected for systems with a weak binding, in analogous to the halo nuclei with neutrons in weak binding observed in nuclear physics [59]. There is the question whether charmonium with such a large separation between $Q$ and $\bar{Q}$ may survive in QGP. The dissociation cross sections for these quarkonia by collision with gluons have been calculated and found to be a function of the gluon collision energy, as shown in Fig. 13 of Ref. [27].

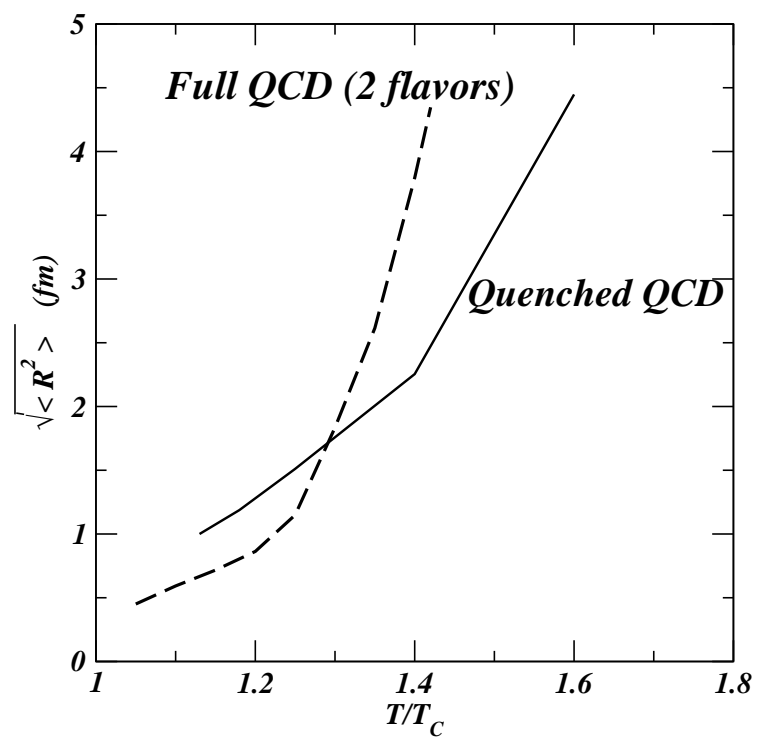

FIG. 4: The root-mean-squared $Q-\bar{Q}$ separation of $L=0$ charmonium as a function of $T / T_{c}$ in quenched QCD (solid curve), and in full QCD with two favors (dashed curve).

From the binding energy of a quarkonium as a function of temperature, one can obtain the temperature at which the quarkonium binding energy vanishes. This is the temperature for the spontaneous dissociation of the quarkonium, as the quarkonium at this temperature will dissociate spontaneously. We list in Table I the heavy quarkonium spontaneous dissociation temperatures calculated with the $W_{1}$ potential, the $F_{1}$ potential, and the $U_{1}$ potential, in quenched QCD.

The $J / \psi$ and $\chi_{b}$ spontaneous dissociation temperatures obtained with the $W_{1}$ potential in quenched QCD are found to be $1.62 T_{c}$ and $1.18 T_{c}$ respectively. Spectral analyses in quenched QCD give the spontaneous dissociation temperature of $1.62-1.70 T_{c}$ for $J / \psi\left[21\right.$ ] and 1.15-1.54T for $\chi_{b}$ 35]. Thus, spontaneous dissociation temperatures obtained with the $W_{1}$ potential agree with those from spectral function analyses. This indicates that the $W_{1}$ potential, defined as the linear combination of $U_{1}$ and $F_{1}$ in Eq. (55), may be the appropriate $Q-\bar{Q}$ potential for studying the stability of heavy quarkonia in quark-gluon plasma.

\section{IX. $Q-\bar{Q}$ POTENTIAL FOR FULL QCD WITH TWO FLAVORS}

The interaction energy between a heavy quark and a heavy antiquark in the color-singlet state in two-flavor full QCD was studied by Kaczmarek and Zantow [34]. In full QCD with 2 flavors, $F_{1}$ and $U_{1}$ can be represented by a color-Coulomb interaction at short distances and a completely screened, constant, potential at large distances as given 
in Ref. [28], although other alternative representations have also been presented [36, 37]. The transitional behavior linking the two different spatial regions can be described by a radius parameter $r_{0}(T)$ and a diffuseness parameter $d(T)$, as in the Wood-Saxon shape potential in nuclear physics,

$$
\left\{F_{1}, U_{1}\right\}(R, T)=-\frac{4}{3} \frac{\alpha_{s}(T)}{R} f(R, T)+C(T)[1-f(R, T)],
$$

where

$$
f(R, T)=\frac{1}{\exp \left\{\left(R-r_{0}(T)\right) / d(T)\right\}+1} .
$$

In principle, it is necessary to specify only the temperature dependence of $F_{1}(R, T)$ as the internal energy $U_{1}(R, T)$ can be obtained from $F_{1}$ and its derivative with respect to $T$. In practice, as Kaczmarek and Zantow [34] have obtained $U_{1}(R, T)$ by a careful numerical differentiation, it is convenient to parametrize the internal energy in the above simple form for practical calculations.

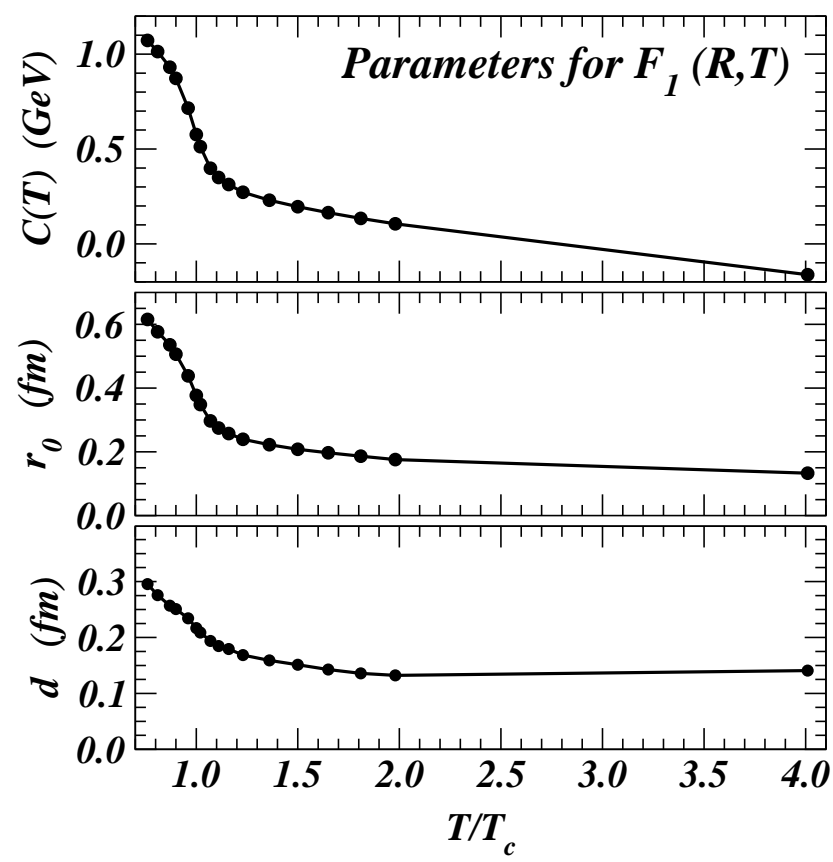

FIG. 5: The parameters $C, r_{0}$, and $d$ for the color-singlet free energy $F_{1}(R, T)$ in two-flavor QCD as given in Eq. (56).

In searching for the coupling constant $\alpha_{s}$ that fits the lattice quantities, we found that the value of $\alpha_{s}$ centers around 0.3. The fit to the lattice gauge quantities does not change significantly whether we allow $\alpha_{s}$ to vary. It is convenient to keep the value of $\alpha_{s}$ to be 0.3 so that there are only three parameters for each temperature.

For the free energy $F_{1}(R, T)$ in two-flavor QCD [34], the set of parameters $C, r_{0}$, and $d$ are shown in Fig. 5, and the corresponding fits to $F_{1}$ are shown in Fig. 6. For the internal energy $U_{1}(R, T)$ in two-flavor QCD [34], the set of parameters $C, r_{0}$, and $d$ are shown in Fig. 7 , and the corresponding fits to the lattice gauge internal energy $U_{1}$ results shown in Fig. 8.

If the thermodynamical quantity $F_{1}$ or $U_{1}$ are treated as a potential, then the quantity $C(T)$ is an approximate measure of the depth of the potential measured from the flat potential surface at large distances relative to the potential well at short distances. For the free energy $F_{1}$, the $C(T)$ parameter has the value of about $1 \mathrm{GeV}$ at $T \sim 0.8 T_{c}$, and it decreases to $0.5 \mathrm{GeV}$ at $T_{c}$. The free energy as a potential will has a well depth of about $0.5 \mathrm{GeV}$ for $T$ close to $T_{c}$ and the well depth decreases to about $0.1 \mathrm{GeV}$ at $T \sim 2 T_{c}$.

One notes that there is a significant change in the slopes of $C(T)$ at $T \sim T_{c}$ for $F_{1}$. As a consequence, the parameter $C(T)$ for $U_{1}$ exhibits a peaks at $T \sim T_{c}$. The transitional radius $r_{0}$ for $F_{1}$ decreases gradually from about $0.6 \mathrm{fm}$ to about $0.15 \mathrm{fm}$ and the diffuseness parameter $d$ decreases slowly from $0.3 \mathrm{fm}$ to about $0.15 \mathrm{fm}$, as temperatures decreases from $0.7 T_{c}$ to $4 T_{c}$. 


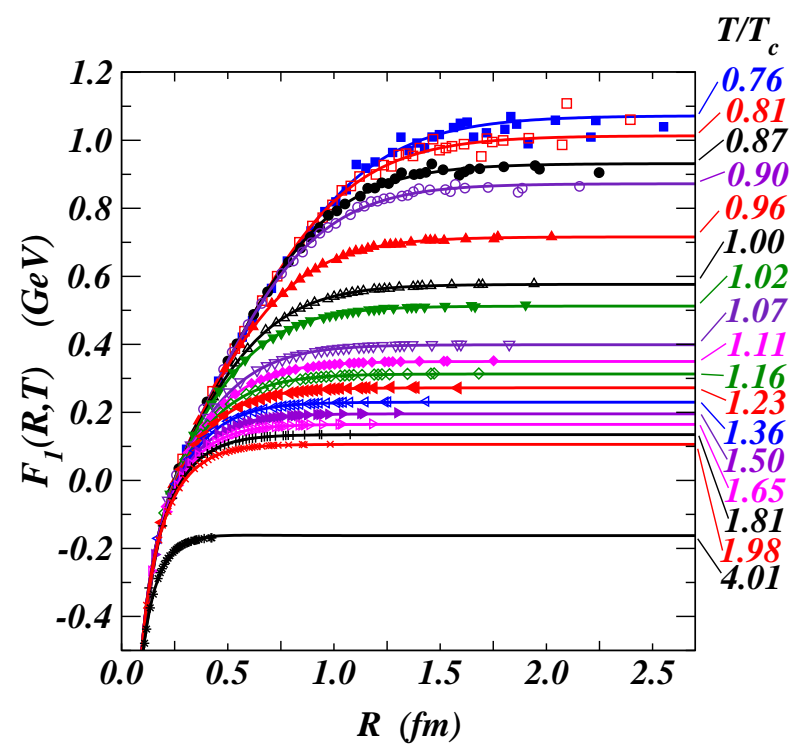

FIG. 6: The symbols represent the color-singlet free energy, $F_{1}(R, T)$, for two-flavor QCD [34], and the curves are the fits using the screened potential, Eq. (56), with parameters given in Fig. 5.

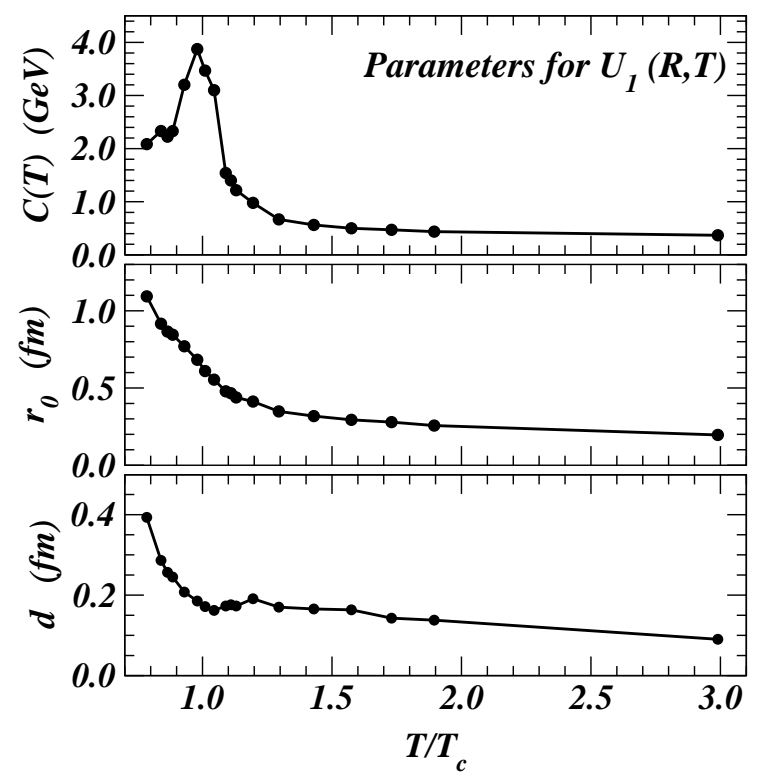

FIG. 7: The parameters $C, r_{0}$, and $d$ for the color-singlet internal energy $U_{1}(\mathbf{R}, T)$ as given in Eq. (56).

For the internal energy $U_{1}$, the parameter $C(T)$ is quite large, attaining the value of about $3 \mathrm{GeV}$ for $T$ close to $T_{c}$. This indicates that if $U_{1}$ is used as a potential, the potential depth at temperatures close to the transition temperature is of order $3 \mathrm{GeV}$, which is a very deep potential indeed. The parameter $C(T)$ decreases to about 0.8 $\mathrm{GeV}$ when the temperature exceeds about $1.5 T_{c}$. The transition radius $r_{0}$ is about $1 \mathrm{fm}$ for $T$ close to $0.8 T_{c}$, and it decreases to about $0.2 \mathrm{fm}$ for $T \sim 4 T_{c}$. The diffuseness parameter $d(T)$ for the internal energy decreases substantially at temperatures below $T_{c}$, but maintains a relatively constant values of 0.1 to $0.2 \mathrm{fm}$ for $T$ greater than $T_{c}$.

The comparison in Fig. 6 and 8 shows that the free energy $F_{1}$ and the internal energy $U_{1}$ with the set of parameters in Figs. 4 and 6, adequately describe the lattice-gauge data and can be used to calculate the eigenvalues and eigenfunctions of heavy quarkonia.

In our description, the $Q-\bar{Q}$ potential is a linear combination of $U_{1}$ and $F_{1}$ with coefficients depending on the equation of state. The equation of state in full QCD with two flavors has been obtained by Karsch et al. [47]. We show their results of $\epsilon / T^{4}$ and $3 p / T^{4}$ in Fig. $9(a)$. The ratio $a(T)=3 p / \epsilon$ and the $U_{1}$ and $F_{1}$ fractions as a function of $T$ are shown in Figs. $8(b)$ and $8(c)$ respectively. Similar to the case of quenched QCD, the $F_{1}$ fraction is close to 


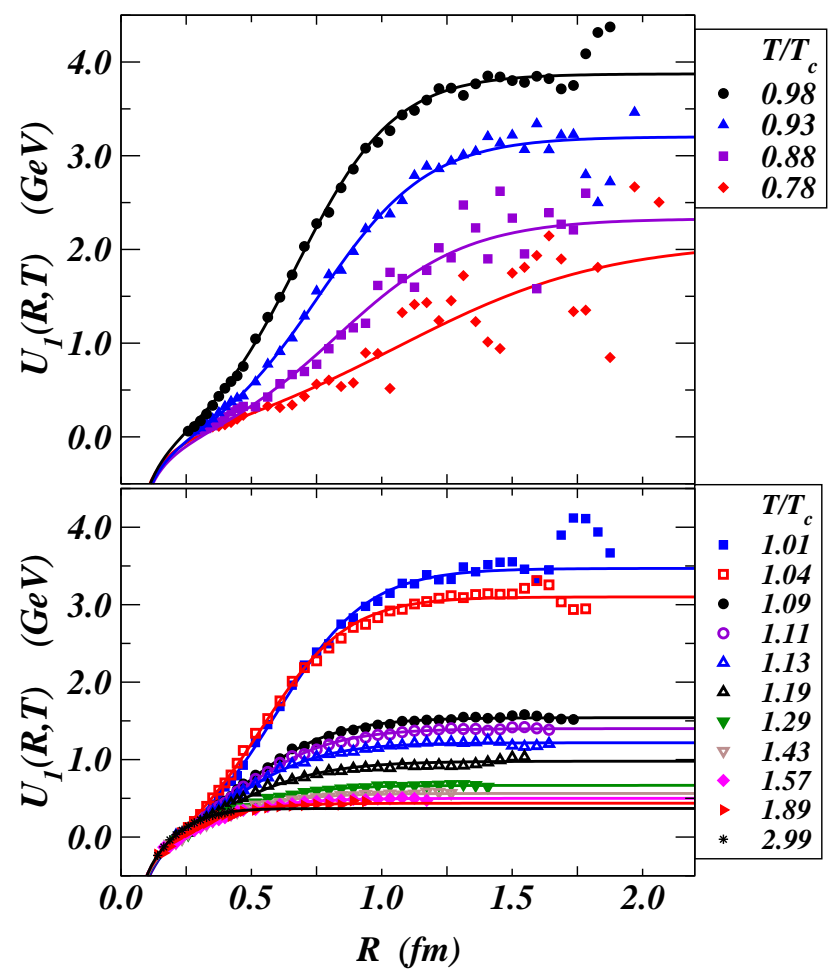

FIG. 8: The symbols represent $U_{1}(R, T)$ for two-flavor QCD obtained by Kaczmarek et al. [34] and the curves are the fits using Eq. (56), with parameters given in Fig. 7 .

unity near $T_{c}$ and it decreases to $3 / 4$ at large temperatures while $U_{1}$ fraction is nearly zero at $T_{c}$ and it increases to about $1 / 4$ at high temperatures. These quantities, together with $F_{1}$ and $U_{1}$ specifies the $Q-\bar{Q}$ potential for bound state calculations.

\section{HEAVY QUARKONIA IN QUARK-GLUON PLASMA}

In full QCD with two flavors, the transition temperature is $T_{c}=202 \mathrm{MeV}$ [34]. To calculate the charmonium energy levels, we employ a quark mass $m_{c}=1.41$ and $m_{b}=4.3 \mathrm{GeV}$.

Energy levels of charmonium states calculated with different potentials in full QCD with two flavors are shown in Fig. 10 as a function of the temperature in units of $T_{c}$. The $J / \psi$ and $\eta_{c}$ states are weakly bound and they dissociate at $1.21 T_{c}$ in the $F_{1}$ potential, at $1.42 T_{c}$ in the $W_{1}$ potential, and at $2.22 T_{c}$ in the $U_{1}$ potential. The $\chi_{c}$ state dissociates below $T_{c}$ in the $F_{1}$ potential, at $1.05 T_{c}$ in the $W_{1}$ potential, and at $1.17 T_{c}$ in the $U_{1}$ potential. At temperatures slightly greater than $T_{c}$, they are weakly bound in the $F_{1}$ potential but are strongly bound in the $U_{1}$ potential, with a binding energy of about $0.7 \mathrm{GeV}$ at $1.1 T_{c}$. The binding of the states in the $W_{1}$ potential lies between these two limits.

The dissociation temperature for $J / \psi$ and $\eta_{c}$ in full QCD with two flavors have been examined in the spectral function analysis by Aarts et al. [60]. As the calculations have been carried out only with a small lattice volume and a small set of statistics, there are possible systematic uncertainties which prevented a precise determination of the pseudocritical temperature. Preliminary results indicate that the $J / \psi$ state may be bound up to about 2.T [60]. More definitive results will await a greater lattice volume and larger statistics.

We also carry out the analysis of bottomium $1 s, 2 p$, and $2 s$ states. Fig. 11 gives the state energies as a function of $T / T_{c}$ for different potentials. The eigenenergies of $\Upsilon$ and $\eta_{b}$ in two-flavor QCD are about $-0.1 \mathrm{GeV}$ at $T=1.1 T_{c}$ in the $F_{1}$ potential, and are about $-1.0 \mathrm{GeV}$ in the $U_{1}$ potential. The eigenenergies in the $W_{1}$ lie in between those of the $F_{1}$ and $U_{1}$ potentials. These states dissociate spontaneously at $2.9 T_{c}$ in the $F_{1}$ potential, 3.40 in the $W_{1}$ potential, and about 4 to $5 T_{c}$ in the $U_{1}$ potential.

In full QCD with two flavors, the $\chi_{b}$ state dissociates at $1.07 T_{c}$ in the $F_{1}$ potential, at 1.22 in the $W_{1}$ potential, and at 1.61 in the $U_{1}$ potential. The $\Upsilon^{\prime}$ and $\eta_{b}^{\prime}$ state dissociates at $1.06 T_{c}$ in the $F_{1}$ potential, at 1.18 in the $W_{1}$ potential, and at 1.47 in the $U_{1}$ potential. 


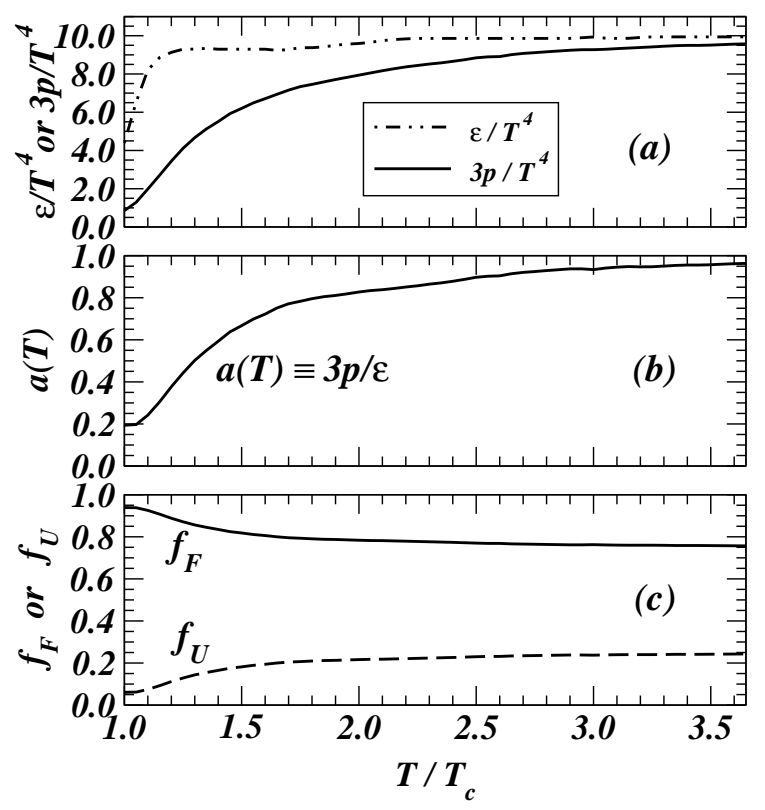

FIG. 9: The equation of state in full QCD with two flavors: $(a)$ the quantity $\epsilon / T^{4}$ and $3 p / T^{4},(b)$ the ratio $a(T)=3 p / \epsilon$, and (c) the $U_{1}$ and $F_{1}$ fractions in the $Q-\bar{Q}$ potential $U_{Q \bar{Q}}^{(1)}$.
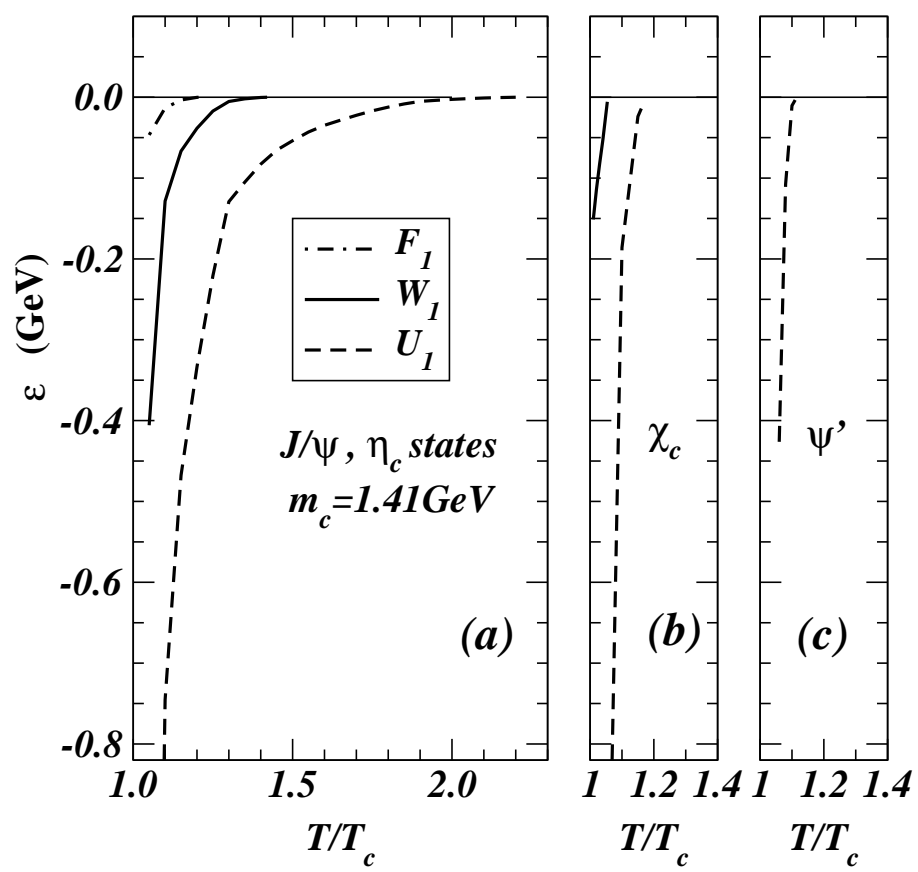

FIG. 10: Energy levels of charmonium in the quark-gluon plasma as a function of temperature calculated with the $F_{1}(R, T)$, $W_{1}(R, T)$, and $U_{1}(R, T)$ potentials in two-flavor QCD. Fig. $10(a)$ is for the $J / \psi$ and $\eta_{c}$ state, Fig. $10(b)$ is for the $\chi_{c}$ state, and Fig. 10(c) is for the $\psi^{\prime}$ state.

In Table I we list the dissociation temperatures of different quarkonia obtained in full QCD with two flavors. A comparison of the dissociation temperatures from the quenched QCD and full QCD with two flavors show the effects of the dynamical quarks. Dynamical quarks increases the degree of screening, but at the same time, lowers the phase transition temperature. They lead to a more diffused potential with a greater screening length. As a consequence, the binding energy of the $1 s$ states is lowered and the dissociation temperature decreases. We can chooses the $W_{1}$ potential as the more appropriate potential for the $Q-\bar{Q}$ pair, as it gives the best agreement with spectral function analysis in quenched QCD. For this $W_{1}$ potential, the dissociation temperature decreases from $1.62 T_{c}$ in quenched 


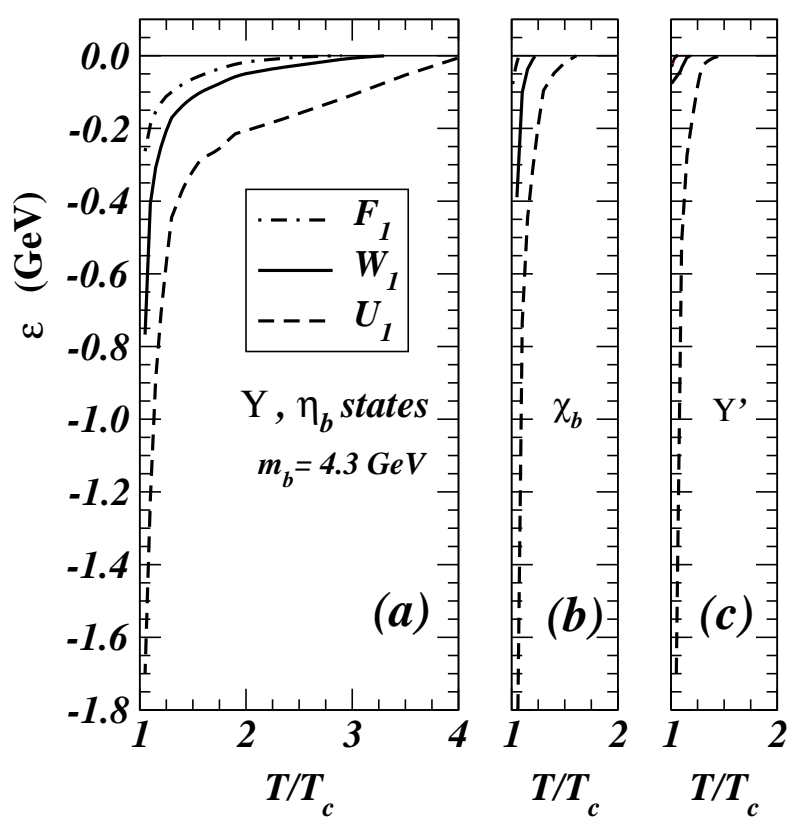

FIG. 11: Energy levels of bottomium in the quark-gluon plasma as a function of the temperature calculated with the $F_{1}(\mathbf{R}, T)$, $W_{1}(\mathbf{R}, T)$, and $U_{1}(R, T)$ potentials in full QCD with two flavors. Fig. 11(a) is for the $\Upsilon$ and $\eta_{b}$ state, Fig. 11(b) is for the $\chi_{b}$ state, and Fig. $11(c)$ is for the $\Upsilon^{\prime}$ state.

QCD to $1.42 T_{c}$ in full QCD with two flavors. The effects of the dynamical quark in full QCD leads to a slightly weaker binding for $J / \psi$ in the plasma. For the $\chi$ states, the effects of the the additional quark screening does not modify the dissociation temperature substantially. The additional screening tends to move the centrifugal barrier for the $l=1$ state to a smaller radial distance with a slightly higher barrier, resulting in a very slight increase in the dissociation temperature.

\section{QUARK-DRIP LINES IN QUARK-GLUON PLASMA}

To examine the stability of a color-singlet $Q-\bar{Q}$ pair, we consider the quark mass $m_{Q}$ as a variable and evaluate the spontaneous dissociation temperature as a function of the reduced mass $\mu_{\text {red }}=m_{Q} m_{\bar{Q}} /\left(m_{Q}+m_{\bar{Q}}\right)$. The quark drip lines calculated with the $F_{1}, W_{1}$, and $U_{1}$ potentials in quenched QCD are shown in Fig. 12. A state is bound in the $\left(T / T_{c}, \mu_{\text {red }}\right)$ space above a drip line and is unbound below the drip line. Spectral function analysis gives the spontaneous dissociation temperature of 1.62-1.70T $T_{c}$ for $J / \psi\left[21,22\right.$, and 1.15-1.54T $T_{c}$ for $\chi_{b}$ [22, 35]. If one takes the charm quark mass to be $1.41 \mathrm{GeV}$ and the bottom quark mass to be $4.3 \mathrm{GeV}$, the spectral function results can be represented by the solid-circle symbols in Fig. 12. They fall on the drip line curves obtained with the $W_{1}$ potential, indicating that the $W_{1}$ is the appropriate $Q-\bar{Q}$ potential to use for bound state problems.

In quenched QCD, however, the quark-gluon plasma is assumed to consist of gluons only. As dynamical quarks may provide additional screening, it is necessary to consider the case with dynamical quarks. Accordingly, we use the $F_{1}, W_{1}$, and $U_{1}$ potentials evaluated in full QCD with 2 flavors [34, 47] to determine the drip lines in Fig. 13. The drip lines for the $U_{1}$ potential lies lower than that of the $W_{1}$, which in turn lies lower than the drip line of the $F_{1}$ potential. In comparison with quenched QCD results, the $1 s$ drip line in full QCD is shifted to lower temperatures while the $1 p$ drip line in full QCD is only slightly modified.

We shall use the results from the $W_{1}$ potential in full QCD with two flavors to discuss the question of quarkonium stability in quark-gluon plasma, as the $W_{1}$ potential has been found to give results in agreement with spectral function analyses in quenched QCD. For heavy quarkonia, results in Table I and Fig. 13 obtained with the $W_{1}$ potential in full QCD with two flavors indicate that $J / \psi, \chi_{c}, \Upsilon, \chi_{b}$, and $\Upsilon^{\prime}$ may be bound in the plasma up to $1.42 T_{c}, 1.05 T_{c}$, $3.40 T_{c}, 1.22 T_{c}$, and $1.18 T_{c}$ respectively.

The variation of the drip lines with the reduced mass allows us to examine the stability of quarkonia containing quarks of various masses. We need to know the effective masses of different quarks in the quark-gluon plasma. Due to its strong interaction with other constituents, a light quark becomes a dressed quasiparticle and acquires a large quasiparticle mass. In the low temperature region where the spontaneous chiral symmetry breaking occurs with 


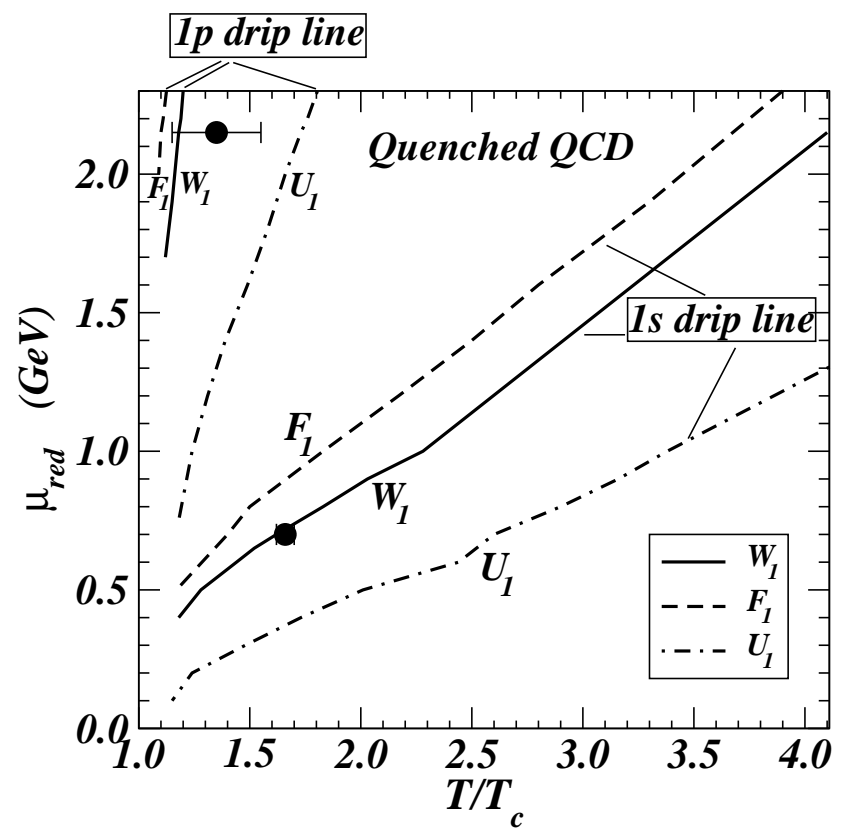

FIG. 12: The quark drip lines in quenched QCD calculated with the $F_{1}, W_{1}$, and $U_{1}$ potentials. The symbols represent results from lattice gauge spectral function analyses.

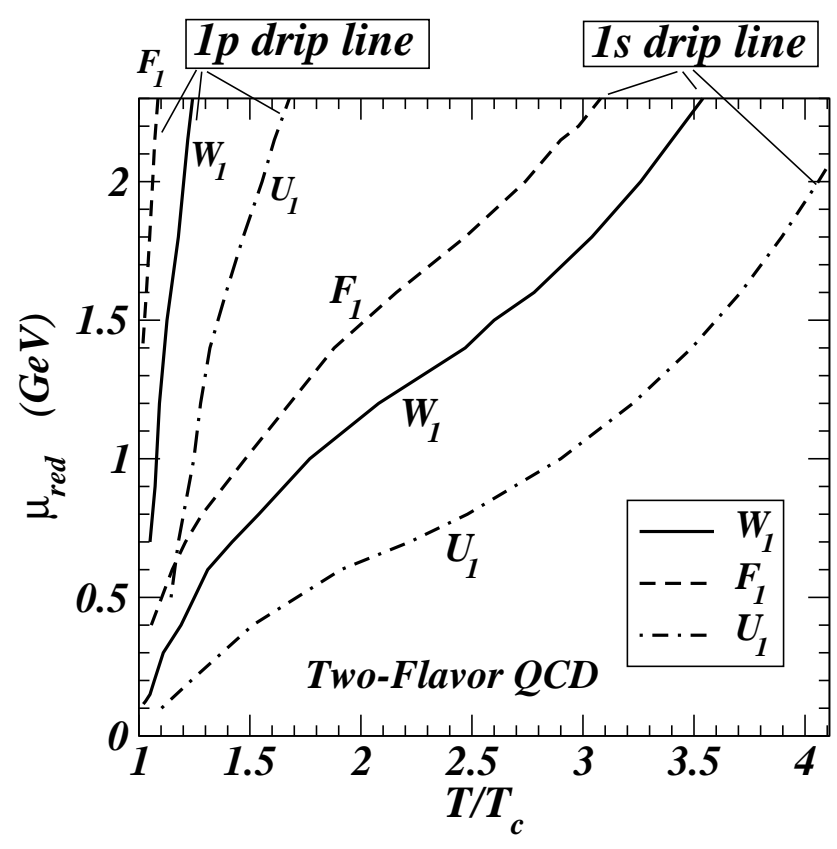

FIG. 13: The quark drip lines in 2-flavor QCD calculated with the $W_{1}, F_{1}$ and $U_{1}$ potentials.

$\langle\bar{\psi} \psi\rangle \neq 0$, the quasiparticle mass is $m_{a} \sim[|g\langle\bar{\psi} \psi\rangle|+$ (current quark mass)], where $g$ is the strong coupling constant and $\langle\bar{\psi} \psi\rangle$ the quark condensate [43, 61, 62]. This quasiparticle mass is the origin of the constituent-quark mass in non-relativistic constituent quark models [43, 62, 63, 64]. In the high temperature perturbative QCD region, the quasiparticle mass is $m_{q} \sim g T / \sqrt{6}$, which is of the order of a few hundred $\mathrm{MeV}$ [65].

As the restoration of chiral symmetry is a second order transition, $\langle\bar{\psi} \psi\rangle$ decreases gradually as the temperature increases beyond $T_{c}$. The light quark quasiparticle mass associated with $\langle\bar{\psi} \psi\rangle$ will likewise decrease gradually from the constituent-quark mass value to the current-quark mass value when the temperature increases beyond $T_{c}$. This tendency for the quasiparticle mass to decrease will be counterbalanced by the opposite tendency for the quasiparticle 'thermal mass' to increase with increasing temperature. As a result of these two counterbalancing tendencies in the 
region of our interest, $T_{c}<T<2 T_{c}$, the effective mass of the light quarks are relatively constant. By examining the effects of the light quark quasiparticle masses on the quark-gluon plasma equation of state, Levai et al. [66], Szabo et al. [67], and Ivanov et al. [68] estimate that $m_{q}$ is about 0.3 to $0.4 \mathrm{GeV}$ at $T_{c}<T<2 T_{c}$. As in the case of $T=0$, where light quarks with a constituent-quark mass of about $350 \mathrm{MeV}$ mimic the effects of chiral symmetry breaking and non-relativistic constituent quark models have been successfully used for light hadron spectroscopy [62, 63], so the large value of the estimated quasiparticle mass (from 0.3 to $0.4 \mathrm{GeV}$ ) may allow the use of a non-relativistic potential model as an effective tool to estimate the stability of light quarkonia at $T_{c}<T<2 T_{c}$. It will be of interest to investigate the relativistic effects $[48,49]$ in the future.

For light quark masses of 0.3 to $0.4 \mathrm{GeV}$, we can estimate from the results in Fig. 13 for the $W_{1}$ potential that as a quarkonium with light quarks has a reduced mass of $0.15-0.2 \mathrm{GeV}$, it may be bound at temperatures below $(1.05-1.07) T_{c}$. An open heavy quarkonium with a light quark and a heavy antiquark or a light antiquark and a heavy quark have a reduced mass of about $0.3-0.4 \mathrm{GeV}$ and may be bound at temperatures below $(1.11-1.19) T_{c}$.

Another lattice gauge calculation gives $m_{q} / T=3.9 \pm 0.2$ at $1.5 T_{c}[69]$, which implies that at $T=1.5 T_{c}$ (or about $0.3 \mathrm{GeV}$ ), the quark mass will be $\sim 1.2 \mathrm{GeV}$ for $(u, d, s)$ quarks. Such a 'light' quark quasiparticle mass appears to be quite large and may be uncertain, as the plasma will have approximately equal abundances of 'light' and charm quarks, which is however not observed. There may also be difficulties in reproducing the plasma equation of state. With this mass, a 'light' quarkonium will have a reduced mass of $0.6 \mathrm{GeV}$, and the quarkonium may be bound at temperatures below $\sim 1.31 T_{c}$.

In either case, the drip lines of Fig. 13 for full QCD with 2 flavors obtained with the $W_{1}$ potential do not support bound $Q \bar{Q}$ states with light quarks beyond $1.5 T_{c}$. A recent study of baryon-strangeness correlations suggests that the quark-gluon plasma contains essentially no bound $Q \bar{Q}$ component at $1.5 T_{c}$ [44].

\section{CONCLUSIONS AND DISCUSSIONS}

The degree to which the constituents of a quark-gluon plasma (QGP) can combine to form composite entities is an important property of the plasma. To study the composite nature of the plasma, we need to examine the stability of quarkonium in quark-gluon plasma which depends on the $Q-\bar{Q}$ potential. We seek to extract the $Q-\bar{Q}$ potential from thermodynamical quantities obtained in lattice gauge calculations. For such a purpose, we need the relationship between the $Q-\bar{Q}$ potential and the internal energy obtained in lattice gauge calculations. Such a relationship was derived previously in Ref. [27]. We would like to gain additional support by examining whether a similar relationship exists between the $Q-\bar{Q}$ potential and the internal energy in an analogous, but not identical, case of Debye screening.

We find that in adiabatic motion of $Q$ and $\bar{Q}$ under Debye screening, (1) the potential for the $Q$ and $\bar{Q}$ in the Schrödinger equation contains the interactions that act on $Q$ and $\bar{Q},(2)$ this $Q-\bar{Q}$ potential under Debye screening is only part of the total internal energy of the system, (3) the other part of the internal energy is the internal energy of the medium particles, and (4) many thermodynamical quantities such as the number, the entropy, and the internal energy of the medium particles increases with the separation between $Q$ and $\bar{Q}$ in the grand canonical ensemble. Therefore, to obtain the Debye screening potential between two static charges, it is necessary to subtract out the internal energy of the medium particles from the total internal energy. These results supports a similar conclusion reached earlier in the analogous lattice gauge theory [27].

We are thus led to obtain the $Q-\bar{Q}$ potential in the quark-gluon plasma by subtracting out the internal energy of the medium particles from the total internal energy in the grand canonical ensemble. We proposed a method to subtracting out the internal energy of the medium by making use of the equation of state of the quark-gluon plasma obtained in an independent lattice gauge calculation [27]. The potential can then be represented as a linear combination of $U_{1}$ and $F_{1}$, with coefficients depending on the quark-gluon plasma equation of state. The proposed potential in the quenched approximation is found to give dissociation temperatures that agree with those from spectral function analyses. It can be generalized to the case of full QCD to discuss quarkonium states in the plasma.

The knowledge of the single-particle states using potentials extracted from lattice gauge calculations in full QCD can then be used to examine the limit of stability of both heavy and light quarkonia and to determine the location of the quark drip lines.

The quark drip lines allows one to ascertain the degree of stability of heavy and light quarkonia when the masses of the quarks are known. $J / \psi, \chi_{c}, \Upsilon, \chi_{b}$, and $\Upsilon^{\prime}$ are found to be stable in the plasma and dissociate at different temperatures. The characteristics of the quark drip lines severely limit the region of possible quarkonium states with light quarks to temperatures close to the phase transition temperature. Various estimates give a light quark mass of about $0.3-0.4 \mathrm{GeV}$ [66, 67, 68], which is not very different from the constituent quark masses in non-relativistic quark models of hadrons. Bound quarkonia with light quarks may exist very near the phase transition temperature if their 
effective quark mass is of the order of $300-400 \mathrm{MeV}$ and higher.

The author thanks Drs. H. Crater and Su-Houng Lee for helpful discussions. This research was supported in part by the Division of Nuclear Physics, U.S. Department of Energy, under Contract No. DE-AC05-00OR22725, managed by UT-Battle, LLC and by the National Science Foundation under contract NSF-Phy-0244786 at the University of Tennessee.

[1] K. Adcox et al, PHENIX Collaboration, Formation of Dense Partonic Matter in Relativistic Nucleus-Nucleus Collisions at RHIC: Experimental Evaluation by the PHENIX Collaboration, nucl-ex/0410003.

[2] J. Adams et al., STAR Collaboration, Experimental and Theoretical Challenges in the Search for the Quark Gluon Plasma: The STAR Collaboration's Critical Assessment of the Evidence from RHIC Collisions, nucl-ex/0501009.

[3] I. Arsene et al., BRAHMS Collaboration, Quark Gluon Plasma an Color Glass Condensate at RHIC? The perspective from the BRAHMS experiment, nucl-ex/0410020.

[4] B. B. Back et al., Phobos Collaboration, The PHOBOS Perspective on Discoveries at RHIC, nucl-ex/0410022.

[5] K. P. Das and R. C. Hwa, Phys. Lett. 68B, 459 (1977); R. C. Hwa, Phys. Rev. D 22, 1593 (1980).

[6] R. C. Hwa and C.B. Yang, Phys. Rev. C70, 024904 (2004).

[7] V. Greco, C. M. Ko, and P. Levai, Phys. Rev. Lett. 90, 202302 (2003).

[8] V. Greco et al., Phys. Rev. C 68, 034904 (2003).

[9] R. J. Fries, B. Muller, C. Nonaka, and S. A. Bass, Phys. Rev. Lett. 90, 202303 (2003); Phys. Rev. C 68, 044902 (2003).

[10] R. J. Fries, S. A. Bass, and B. Müller, Phys. Rev. Lett. 94, 122301 (2005).

[11] P. Braun-Munzinger, J. Stachel, J.P. Wessels, and N. Xu, Phys. Lett. B 344, 43 (1995).

[12] P. Braun-Munzinger, J. Stachel, J. P. Wessels, and N. Xu, Phys. Lett. B365, 1 (1996).

[13] J. Cleymans and K. Redlich, Nucl.Phys. A661, 379 (1999).

[14] P. Braun-Munzinger and J. Stachel, Nucl. Phys. A606, 320 (1996).

[15] J. Cleymans and K. Redlich, Phys. Rev. C60, 054908 (1999).

[16] J. Cleymans, H. Oeschler, and K. Redlich, J. Phys. G25, 281 (1999).

[17] P. Braun-Munzinger and J. Stachel, J. Phys. G28, 1971 (2002).

[18] P. Braun-Munzinger, J. Stachel, and C. Wetterich, Phys. Lett. B596, 61 (2004).

[19] P. Braun-Munzinger, K. Redlich, and J. Stachel, nucl-th/0304013, in Quark Gluon Plasma 3, (eds.) R. Hwa and X.N. Wang.

[20] J. Cleymans, H. Oeschler, K. Redlich, and S. Wheaton, Phys. Lett. B615 , 50 (2005).

[21] M. Asakawa, T. Hatsuda, and Y. Nakahara Nucl. Phys. A715, 863 (2003); M. Asakawa and T. Hatsuda, Phys. Rev. Lett. 92, 012001 (2004); T. Hatsuda, hep-ph/0509306

[22] S. Datta, F. Karsch, P. Petreczky, and I. Wetzorke, Phys. Rev. D69, 094507 (2004) and J. Phys. G31, S351 (2005).

[23] E. V. Shuryak and I. Zahed, Phys. Rev. C70, 021901(R) (2004); Phys. Rev. D70, 054507 (2004); E. V. Shuryak, Nucl. Phys. A750, 64 (2005).

[24] G. E. Brown, C. H. Lee, M. Rho, and E. Shuryak, Nucl. Phys. A 740, 171 (2004).

[25] T. Matsui and H. Satz, Phys. Lett. B178, 416 (1986).

[26] C. Y. Wong, Phys. Rev. C 65, 034902 (2002); J. Phys. G28, 2349 (2002).

[27] C. Y. Wong, Phys. Rev. C72, 034906 (2005).

[28] C. Y. Wong, in Proceedings of XVIIth Recontre de Blois, Chateau de Blois, France, May 15-20, 2005, edited by M. Haguenauer, B. Nicolescu, and J. T. Thanh Van, p. 467, hep-ph/0509088

[29] C. Y. Wong, J. Phys. G32, S301 (2006).

[30] C. Y. Wong and H. W. Crater, Phys. Rev. D75, 034505 (2007).

[31] S. Digal, P. Petreczky, and H. Satz, Phys. Lett. B514, 57 (2001); Phys. Rev. D64, 094015 (2001).

[32] O. Kaczmarek, F. Karsch, and P. Petreczky, and F. Zantow, Phys. Lett. B543, 41 (2002); F. Zantow, O. Kaczmarek, F. Karsch, and P. Petreczky, hep-lat/0301015.

[33] O. Kaczmarek, F. Karsch, P. Petreczky, and F. Zantow hep-lat/0309121.

[34] O. Kaczmarek and F. Zantow, Phys. Rev. D71, 114510 (2005), and O. Kaczmarek and F. Zantow, hep-lat/0506019.

[35] K. Petrov, Eur. Phys. J. C43, 67 (2005); A. Mocsy, Talk presented at Quark Matter Conference, 2005, hep-ph/0510135

[36] S. Digal, O. Kaczmarek, F. Karsch, and H. Satz, Eur. Phys. J. C43, 71 (2005).

[37] W.M. Alberico, A. Beraudo, A. De Pace, and A. Molinari, hep-ph/0507084;

[38] D. Blaschke, O. Kaczmarek, E. Laermann, and V. Yudichev, Eur. Phys. J. C43, 81 (2005).

[39] T. Song and S. H. Lee, Phys. Rev. D 72, 034002 (2005).

[40] C. DeTar, Phys. Rev. D 32, 276 (1985); C. DeTar, Phys. Rev. D 37, 2328 (1988).

[41] T. H. Hansson, S. H. Lee, and I. Zahed Phys. Rev. D 37, 2672 (1988).

[42] Yu. A. Simonov, Phys. Atom. Nucl. 58, 309 (1995). Yad. Fiz. 58N2, 357 (1995); Yu.A.Simonov, Phys. Lett. B619 293 (2005).

[43] T. Hatsuda and T. Kunihiro, Phys. Rev. Lett. 55, 158 (1985).

[44] V. Koch, A. Majumder, and J. Randrup, Phys. Rev. Lett. 95, 182301 (2005).

[45] F. Karsch, S. Ejiri, and K. Redlich, Nucl. Phys. A774, 619 (2006). 
[46] F. G. Werner and J. A. Wheeler, Phys. Rev. 106, 126 (1958).

[47] F. Karsch, E. Laermann, and A. Peikert, Phys. Lett. B478, 447 (2000); F. Karsch, Nucl.Phys. A698, 199 (2002).

[48] H. W. Crater and P. Van Alstine, Phys. Rev. D70, 034026, (2004).

[49] H. W. Crater, C. Y. Wong, and P. Van Alstine, Phys. Rev. D74, 054028 (2006).

[50] A. Mocsy and P. Petreczky, Phys. Rev. D73, 074007 (2006).

[51] A. Mocsy and P. Petreczky, J. Phys. G32, S515 (2006).

[52] L. D. Landau, Izv. Akad. Nauk. SSSR. ser. fiz. 17, 51 (1953); L. D. Landau and S. Z. Belenkii, Usp. Fiz. Nauk 56, 309 (1955).

[53] L. D. Landau and L. M. Lifshitz, Statistical Physics, Pergamon Press, Oxford, 1959, Chapter I.

[54] G. Bali, K. Schilling, and A. Wachter, Phys. Rev. D56 2566 (1997).

[55] N. Ishii, S. Aoki, and T. Hatsuda, hep-lat/0610002

[56] T. Takahashi, T. Doi, and H. Suganuma, hep-lat/0601006 and T. Doi, T. Takahashi, and H. Suganuma, hep-lat/0601008

[57] W. Detmold, K. Orginos, and M. J. Savage, hep-lat/0703009

[58] G. Boyd et al., Nucl. Phys. B 469, 419 (1996)

[59] I. Tanihata, Prog. Part. Nucl. Phys. 35, 505 (1995); V. Rotival and T. Duguet, nucl-th/0702050

[60] G. Aarts, C. Allton, R. Morrin, A. O Cais, M. B. Oktay, M. Peardon, J.-I. Skullerud, Nucl.Phys.Proc.Suppl. 153 296, (2006) [ hep-lat/0511028.

[61] Y. Nambu and G. Jona-Lasinio, Phys. Rev. 122, 345 (1961), and 124, 246 (1961).

[62] A. P. Szczepaniak1 and E. S. Swanson, Phys. Rev. Lett. 87, 072001 (2001).

[63] T. Barnes and E. S. Swanson, D46, 131 (1992).

[64] C. Y. Wong, T. Barnes and E. S. Swanson, Phys. Rev. C65, 014903 (2002).

[65] H. A. Weldon, Phys. Rev. D26, 2789 (1982); K. Kajantie, M. Laine, K. Rummukainen, and Y. Schröder, Phys. Rev. D67, 105008 (2003); J. P. Blaizot, E. Iancu, and A. Rebhan, Phys. Rev. D68, 025011 (2003).

[66] P. Levai and U. Heinz, Phys. Rev. C57, 1879 (1998);

[67] K. K. Szabo and I. Toth, JHEP 0306008 (2003).

[68] Yu. B. Ivanov, V.V. Skokov, and Toneev, Phys. Rev. D71, 014005 (2005).

[69] P. Petreczky, F. Karsch, E.Laemann, S. Stickan, and I. Wetzorke, Nucl. Phys. B, Proc. Suppl. 106, 513 (2002). 